Noname manuscript No.

(will be inserted by the editor)

\title{
A fresh CP look at mixed-binary QPs: New formulations and relaxations
}

\author{
Immanuel M. Bomze • Jianqiang Cheng • \\ Peter J.C. Dickinson • Abdel Lisser
}

Received: date / Accepted: date

\begin{abstract}
Triggered by Burer's seminal characterization from 2009, many copositive (CP) reformulations of mixed-binary QPs have been discussed by now. Most of them can be used as proper relaxations, if the intractable co(mpletely )positive cones are replaced by tractable approximations. While the widely used approximation hierarchies have the disadvantage to use positive-semidefinite (psd) matrices of orders which rapidly increase with the level of approximation, alternatives focus on the problem of keeping psd matrix orders small, with the aim to avoid memory problems in the interior point algorithms. This work continues this approach, proposing new reformulations and relaxations. Moreover, we provide a thorough comparison of the respective duals and establish a monotonicity relation among their duality gaps. We also identify sufficient conditions for strong duality/zero duality gap in some of these formulations and generalize some of our observations to general conic problems.
\end{abstract}

Keywords Copositivity · Completely positive · Conic optimization · Quadratic optimization · Reformulations · Nonlinear optimization · Nonconvex optimization

Mathematics Subject Classification (2000) 90C20 - 90C26 · 90C30

\section{Introduction}

1.1 Motivation and basic ideas

Copositive optimization (or copositive programming coined in [6], abbreviated CP) is a special case of conic optimization, which consists of minimizing a linear function over a (convex) cone subject to additional (inhomogeneous) linear (inequality or equality) constraints. This problem class has a close connection to that of quadratic optimization, which represents the simplest class of hard problems in continuous optimization [18] to minimize a (possibly indefinite) quadratic form over a polyhedron given in standard form:

$$
\min \left\{\mathrm{x}^{\top} \mathrm{Qx}: \mathrm{Ax}=\mathrm{b}, \mathrm{x} \in \mathbb{R}_{+}^{n}\right\}
$$

Immanuel M. Bomze

Institut für Statistik und Operations Research (ISOR),

Universität Wien, 1090 Wien, Oskar-Morgenstern-Platz 1, Austria.

E-mail: immanuel.bomze@univie.ac.at

Jianqiang Cheng

Department of Systems and Industrial Engineering, University of Arizona, Tucson, AZ 85721, USA.

E-mail: jqcheng@email.arizona.edu

Peter J.C. Dickinson

Dep. Appl. Mathematics, University of Twente, P.O. Box 217, 7500 AE Enschede, The Netherlands.

E-mail: p.j.c.dickinson@utwente.nl

Abdel Lisser

Laboratoire de Recherche en Informatique (LRI),

Université Paris Sud - XI Bât. 650, 91405 Orsay Cedex, France

E-mail: lisser@lri.fr 
By lower-case sans-serif letters we denote vectors in $n$-dimensional Euclidean space $\mathbb{R}^{n}$ (e.g. the zero vector o), by upper-case sans-serif letters matrices (e.g. the zero matrix O), and by ${ }^{\top}$ transposition. The nonnegative orthant is denoted by $\mathbb{R}_{+}^{n}:=\left\{\mathrm{x} \in \mathbb{R}^{n}: x_{i} \geq 0\right.$ for all $\left.i\right\}$. In most cases, $x_{i}$ refers to the $i$-th coordinate of $\mathrm{x}$, but sometimes we need to refer to coordinates of more complicated expressions like $\mathrm{Ax}$ or $\mathrm{x}_{k}$ etc., which will then be denoted by subscripts of parentheses, i.e. $(\mathrm{Ax})_{i}$ or $\left(\mathrm{x}_{k}\right)_{i}$ etc.

The basic lifting idea goes back to Shor 22] and linearizes the quadratic form $\mathrm{x}^{\top} \mathrm{Qx}=\operatorname{trace}\left(\mathrm{x}^{\top} \mathrm{Q} \mathrm{x}\right)=$ $\operatorname{trace}\left(\mathrm{Q} x \mathrm{x}^{\top}\right)=\left\langle\mathrm{Q}, \mathrm{xx^{ \top } \rangle}\right.$ by introducing the new symmetric matrix variable $\mathrm{X}=\mathrm{x} \mathrm{x}^{\top}$ and using the Frobenius inner product $\langle\mathrm{X}, \mathrm{Y}\rangle=\operatorname{trace}(\mathrm{XY})=\sum_{i, j=1}^{n} X_{i j} Y_{i j}$. Squaring the linear constraints in (1), we arrive in a similar way at linear constraints of the form $\left\langle\mathrm{A}_{i}, \mathrm{X}\right\rangle=b_{i}^{2}$, where $\mathrm{A}_{i}=\mathrm{a}_{i} \mathrm{a}_{i}^{\top}$ and $\mathrm{a}_{i}^{\top}$ is the $i$-th row of $\mathrm{A}$. hull

Now the set of all these $X=x x^{\top}$ generated by nonnegative $x$ is non-convex since rank $\left(x x^{\top}\right)=1$. The convex

$$
\operatorname{conv}\left\{x x^{\top}: x \in \mathbb{R}_{+}^{n}\right\}
$$

results in a convex matrix cone called the cone of completely positive matrices (see e.g. 3]). Note that a similar construction dropping nonnegativity constraints leads to

$$
\operatorname{conv}\left\{x x^{\top}: x \in \mathbb{R}^{n}\right\}
$$

the cone of positive-semidefinite matrices, the basic set in Semidefinite Optimization (SDP), wherefrom the above lifting idea was borrowed.

Both variants of lifting give rise to relaxations which are considerably tighter than previously used ones. But one can say more: Burer [10] showed in a seminal paper that the CP approach is in fact a reformulation rather than a relaxation, of any mixed-binary QP, under mild conditions (for surveys see e.g. [4, 12, 13, 16]). Since then, many alternative CP reformulations of mixed-binary QPs have been discussed, and one of the aims of this article is a unified view of them. We will observe that although the primal CP problems are all identical, their relaxations and their duals (and the inner approximation of the duals) may differ, which is important in practical implementations.

In this paper we will relate the reformulations of Burer [10] with some more recent reformulations inspired by 1, 11, 14, and complement these with some new reformulations in Section 2 Furthermore, in Section 3 we will deal in detail with their respective dual problems and establish a monotonicity relation among their duality gaps. Finally we look at conditions guaranteeing strong duality/zero duality gap. Note that in an effort to reduce notation, we use min and max at places where we could write inf and sup if attainability is not (yet) guaranteed.

\subsection{Further notation and terminology}

For two integers $m$ and $n$ with $m \leq n$ we abbreviate $[m: n]$ for the integer interval $\{m, m+1, \ldots, n\}$. Given an optimization problem $(\mathrm{P})$, we denote by feas $(\mathrm{P})$ the feasible set of this problem.

The set of all $d \times p$ matrices is denoted by $\mathbb{R}^{d \times p}$, the subset of those with no negative entries by $\mathbb{R}_{+}^{d \times p}$, and

$$
\mathcal{S}^{d}:=\left\{\mathrm{X} \in \mathbb{R}^{d \times d}: \mathrm{X}=\mathrm{X}^{\top}\right\}
$$

while

$$
\mathcal{N}^{d}:=\mathcal{S}^{d} \cap \mathbb{R}_{+}^{d \times d}=\left\{\mathrm{X} \in \mathcal{S}^{d}: X_{i j} \geq 0 \text { for all } i, j\right\} .
$$

By $_{n} \in \mathcal{S}^{n}$ we denote the identity matrix with columns $\mathrm{e}_{i} \in \mathbb{R}^{n}, i \in[1: n]$.

As already mentioned, the Frobenius inner product is denoted by $\langle\mathrm{S}, \mathrm{X}\rangle=\operatorname{trace}(\mathrm{SX})$, where $\{\mathrm{S}, \mathrm{X}\} \subset \mathcal{S}^{d}$. With regard to any duality, for a given cone $\mathcal{A}$, we consider its dual cone

$$
\mathcal{A}^{*}=\{\mathrm{b}:\langle\mathrm{a}, \mathrm{b}\rangle \geq 0 \text { for all } \mathrm{a} \in \mathcal{A}\} .
$$

We let $\mathcal{S}_{+}^{d}$ be the positive-semidefinite (psd) cone, i.e. the set of matrices $X \in \mathcal{S}^{d}$ such that $X$ is positivesemidefinite (psd):

$$
\mathcal{S}_{+}^{d}:=\operatorname{conv}\left\{\mathrm{xx}^{\top}: \mathrm{x} \in \mathbb{R}^{d}\right\}
$$

It is well known that this cone is self-dual while there are other important cones which are not. For instance, the following two cones are dual to each other: the cone of all symmetric $d \times d$ copositive matrices denoted by

$$
\mathcal{C O} \mathcal{P}^{d}:=\left\{\mathrm{M} \in \mathcal{S}^{d}: \mathrm{x}^{\top} \mathrm{Mx} \geq 0 \text { for all } \mathrm{x} \in \mathbb{R}_{+}^{d}\right\}
$$


is the dual cone of $\mathcal{C P}^{d}$, the cone of all completely positive matrices of order $d$ :

$$
\mathcal{C} \mathcal{P}^{d}:=\operatorname{conv}\left\{\mathrm{xx}^{\top}: \mathrm{x} \in \mathbb{R}_{+}^{d}\right\}=\left\{\mathrm{X} \in \mathcal{S}^{d}: \mathrm{X}=\sum_{i=1}^{p(d)} \mathrm{f}_{i} \mathrm{f}_{i}^{\top} \text { for some } \mathrm{f}_{i} \in \mathbb{R}_{+}^{d}\right\} .
$$

Here the upper bound $p(d)$ on the necessary number of summands for $\mathbf{X}$, the so-called $c p$-rank of $\mathbf{X}$, can be set to $p(d)=\max \left\{\left(\begin{array}{c}d+1 \\ 2\end{array}\right)-4, d\right\}$ which is asymptotically tight for large $d[8,21]$.

\subsection{Setup and new variants of CP formulation}

In this paper we consider the following mixed-binary quadratic optimization problem:

$$
\begin{aligned}
& \min x^{\top} Q x+2 c^{\top} x \\
& \text { s.t. } \mathrm{a}_{i}^{\top} \mathrm{x}=b_{i} \quad \text { for } i \in[1: m] \\
& \mathrm{x} \in \mathbb{R}_{+}^{n} \\
& x_{j} \in\{0,1\} \quad \text { for } j \in B,
\end{aligned}
$$

where $B \subseteq[1: n] ; \mathrm{Q} \in \mathcal{S}^{n} ; \mathrm{b} \in \mathbb{R}^{m} ;$ and $\left\{\mathrm{c}, \mathrm{a}_{1}, \ldots, \mathrm{a}_{m}\right\} \subset \mathbb{R}^{n}$, with $\mathrm{a}_{1}, \ldots, \mathrm{a}_{m}$ being linearly independent (otherwise we can remove redundant linear constraints, reducing $m$ ).

We define the polyhedron

$$
\mathcal{Z}:=\left\{x \in \mathbb{R}_{+}^{n}: \mathrm{a}_{i}^{\top} \mathrm{x}=b_{i}, i \in[1: m]\right\}
$$

containing feas $(\mathrm{P})$. We shall then assume there exists $\mathrm{x}_{0} \in \mathcal{Z}$ such that $\left(\mathrm{x}_{0}\right)_{j}>0$ for all $j \in[1: n]$. If this is not the case then either $\mathcal{Z}=\emptyset$ (and so $(\mathrm{P})$ is infeasible) or there exists a $j \in[1: n]$ such that $x_{j}=0$ for all $\mathrm{x} \in \mathcal{Z}$ (and thus also for all $\times$ feasible in $(\vec{P})$ ). In this second case we can discard the variable $x_{j}$ from problem $(\mathrm{P})$, reducing the size of the problem. The checks required in this paragraph can all be performed with simple linear optimization problems. To be more precise, we consider the $n$ LPs $\max _{\mathrm{x} \in \mathcal{Z}} x_{j}$ across all $j \in[1: n]$, stop the optimization early for these LPs (if they are feasible at all) whenever a feasible solution $\mathrm{x}_{j}$ with $\left(\mathrm{x}_{j}\right)_{j}>0$ is encountered, and define $\mathrm{x}_{0}=\frac{1}{n} \sum_{j} \mathrm{x}_{j} \in \mathcal{Z}$.

We shall also assume that the following key assumption holds for this problem (cf. the discussion in [7]):

$$
v \in \mathcal{Z} \Longrightarrow v_{j} \leq 1 \text { for all } j \in B \text {. }
$$

This assumption can always be made to hold by adding slack variables. Letting $\mathcal{Z}_{\infty}$ be the recession cone of $\mathcal{Z}$, i.e. $\mathcal{Z}_{\infty}:=\left\{z \in \mathbb{R}^{n}: x+\lambda z \in \mathcal{Z}\right.$ for all $\left.x \in \mathcal{Z}, \lambda \geq 0\right\}=\left\{z \in \mathbb{R}_{+}^{n}: a_{i}^{\top} z=0, i \in[1: m]\right\}$, the key assumption implies the following property (by looking at $\mathrm{v}=\mathrm{x}_{0}+t z$ with $t \uparrow \infty$ ):

$$
\mathrm{z} \in \mathcal{Z}_{\infty} \quad \Longrightarrow \quad z_{j}=0 \text { for all } j \in B
$$

We shall let $\left\{\mathrm{a}_{m+1}, \ldots, \mathrm{a}_{n}\right\} \subset \mathbb{R}^{n}$ be such that $\mathrm{a}_{m+1}, \ldots, \mathrm{a}_{n}$ are linearly independent vectors with $\mathrm{a}_{i}^{\top} \mathrm{a}_{m+j}=$ 0 for all $i \in[1: m]$ and all $j \in[1: n-m]$. Determination of these $\mathbf{a}_{m+1}, \ldots, \mathrm{a}_{n}$ can be done efficiently by complete solution of the orthogonal complement $\left\{\mathrm{a}_{1}, \ldots, \mathrm{a}_{m}\right\}^{\perp}$ by standard linear algebra procedures, such as the singular value decomposition which can be computed within $O\left(\mathrm{~nm}^{2}\right)$ time [23].

Then letting $\mathrm{R} \in \mathbb{R}^{(n+1) \times(n+1-m)}$ be such that

$$
\mathrm{R}=\left[\begin{array}{ccccc}
1 & 0 & 0 & \cdots & 0 \\
x_{0} & a_{m+1} & a_{m+2} & \cdots & a_{n}
\end{array}\right]
$$

we have

$$
\left\{\left(\begin{array}{l}
\zeta \\
\mathrm{z}
\end{array}\right) \in \mathbb{R}^{n+1}: \mathrm{a}_{i}^{\top} \mathrm{z}=b_{i} \zeta \text { for } i \in[1: m]\right\}=\left\{\mathrm{Ry}: \mathrm{y} \in \mathbb{R}^{n+1-m}\right\}
$$

Note that linear independence of $\mathrm{a}_{m+1}, \ldots, \mathrm{a}_{n}$ implies that $\mathrm{R}$ has full column rank, and thus for any $\mathrm{Y} \in \mathcal{S}^{n+1-m}$ we have

$$
\begin{aligned}
\mathrm{RYR}^{\top} \in \mathcal{S}_{+}^{n+1} & \Longleftrightarrow \mathrm{Y} \in \mathcal{S}_{+}^{n+1-m}, \quad \text { and } \\
\mathrm{RYR}^{\top}=0 & \Longleftrightarrow \mathrm{Y}=0 .
\end{aligned}
$$


In [10], Burer showed that provided the key assumption (2) holds, then $(\mathrm{P})$ has the same optimal value as the problem $\mathrm{CPP}$ below.

$$
\begin{aligned}
& \min \langle Q, X\rangle+2 c^{\top} x \\
& \text { s.t. } \mathrm{a}_{i}^{\top} \mathrm{x}=b_{i} \quad \text { for } i \in[1: m] \\
& \left\langle\mathrm{a}_{i} \mathrm{a}_{i}^{\top}, \mathrm{X}\right\rangle=b_{i}^{2} \quad \text { for } i \in[1: m] \\
& X_{j j}=x_{j} \quad \text { for } j \in B \\
& \left(\begin{array}{ll}
1 & \mathrm{x}^{\top} \\
\mathrm{x} & \mathrm{X}
\end{array}\right) \in \mathcal{C P}^{n+1} \text {. }
\end{aligned}
$$

In order to show this, he also proved the result

$$
\left\{\left(\begin{array}{cc}
1 & x^{\top} \\
x & x
\end{array}\right):(x, X) \in \text { feas }[\mathrm{CPP})\right\}=\operatorname{conv}\left\{\left(\begin{array}{l}
1 \\
x
\end{array}\right)\left(\begin{array}{l}
1 \\
x
\end{array}\right)^{\top}: x \in \text { feas }(\mathrm{P})\right\}+\operatorname{conv}\left\{\left(\begin{array}{l}
0 \\
z
\end{array}\right)\left(\begin{array}{l}
0 \\
z
\end{array}\right)^{\top}: z \in \mathcal{Z}_{\infty}\right\}
$$

In the sequel we shall study two alternatives which are equivalent to $\mathrm{CPP}$. By 'equivalent' we mean that their feasible sets are the same, and they have the same objective functions. Hence the following two problems present alternative $\mathrm{CP}$ reformulations of the problem $(\mathrm{P})$. The first reformulation was put forward by [1]

$$
\begin{aligned}
& \min \langle Q, X\rangle+2 c^{\top} x \\
& \text { s.t. } \sum_{i=1}^{m}\left(\mathrm{a}_{i}^{\top} \mathrm{Xa}_{i}-2 b_{i} \mathrm{a}_{i}^{\top} \mathrm{x}+b_{i}^{2}\right)=0 \\
& \sum_{j \in B}\left(X_{j j}-x_{j}\right)=0 \\
& \left(\begin{array}{cc}
1 & x^{\top} \\
x & X
\end{array}\right) \in \mathcal{C} \mathcal{P}^{n+1}
\end{aligned}
$$

along with its doubly-nonnegative (DNN) relaxation,

$$
\begin{aligned}
\min & \langle\mathrm{Q}, \mathrm{X}\rangle+2 \mathrm{c}^{\top} \mathrm{x} \\
\text { s.t. } & \sum_{i=1}^{m}\left(\mathrm{a}_{i}^{\top} \mathrm{Xa}_{i}-2 b_{i} \mathrm{a}_{i}^{\top} \mathrm{x}+b_{i}^{2}\right)=0 \\
& \sum_{j \in B}\left(X_{j j}-x_{j}\right)=0 \\
& \left(\begin{array}{c}
1 \\
\mathrm{x}^{\top} \\
\mathrm{x}
\end{array}\right) \in \mathcal{N}^{n+1} \cap \mathcal{S}_{+}^{n+1} .
\end{aligned}
$$

While the second reformulation is inspired by [11,14], it was apparently not yet put forward in the published literature:

$$
\begin{array}{ll}
\min & \langle\mathrm{Q}, \mathrm{X}\rangle+2 \mathrm{c}^{\top} \mathrm{x} \\
\text { s.t. } & \sum_{j \in B}\left(X_{j j}-x_{j}\right)=0 \\
& \mathrm{RYR}^{\top}=\left(\begin{array}{cc}
1 & \mathrm{x}^{\top} \\
\mathrm{x} & \mathrm{X}
\end{array}\right) \\
& \left(\begin{array}{ll}
1 & \mathrm{x}^{\top} \\
\mathrm{x} & \mathrm{X}
\end{array}\right) \in \mathcal{C} \mathcal{P}^{n+1}, \mathrm{Y} \in \mathcal{S}^{n+1-m}
\end{array}
$$

We will also consider the following new relaxation of this:

$$
\begin{array}{ll}
\min & \langle\mathrm{Q}, \mathrm{X}\rangle+2 \mathrm{c}^{\top} \mathrm{x} \\
\text { s.t. } & \sum_{j \in B}\left(X_{j j}-x_{j}\right)=0 \\
& \mathrm{RYR}^{\top}=\left(\begin{array}{cc}
1 & \mathrm{x}^{\top} \\
\mathrm{x} & \mathrm{X}
\end{array}\right) \\
& \left(\begin{array}{cc}
1 & \mathrm{x}^{\top} \\
\mathrm{x} & \mathrm{X}
\end{array}\right) \in \mathcal{N}^{n+1}, \mathrm{Y} \in \mathcal{S}_{+}^{n+1-m} .
\end{array}
$$

Actually, the formulation in [1] involved one more variable $x_{0}$ in the north west corner of $\left(\begin{array}{ll}1 & x^{\top} \\ x & x\end{array}\right)$, and one more constraint $x_{0}=1$ (see below). It is a matter of implementation whether to keep this equation as a constraint for the optimization process or to use it to fix the variable $x_{0}$ (and discard it). Whatever the choice may be, inspired by the work done in [1,11] and [14, Section 2.1], we shall consider the following four linear subspaces in $\mathcal{S}^{n+1}$ related to the linear constraints of $(\mathrm{P})$ :

$$
\mathcal{L}_{1}=\left\{\left(\begin{array}{cc}
x_{0} & \mathrm{x}^{\top} \\
\mathrm{x} & \mathrm{X}
\end{array}\right) \in \mathcal{S}^{n+1}: \exists \mathrm{Y} \in \mathcal{S}^{n+1-m} \text { s.t. } \mathrm{RYR}^{\top}=\left(\begin{array}{cc}
x_{0} & \mathrm{x}^{\top} \\
\mathrm{x} & \mathrm{X}
\end{array}\right)\right\}=\mathrm{RS}^{n+1-m} \mathrm{R}^{\top}
$$




$$
\begin{aligned}
& \mathcal{L}_{2}=\left\{\left(\begin{array}{cc}
x_{0} \mathrm{x}^{\top} \\
\mathrm{x} & \mathrm{x}
\end{array}\right) \in \mathcal{S}^{n+1}: \begin{array}{l}
\mathrm{a}_{i}^{\top} \mathrm{x}=b_{i} x_{0} \text { for } i \in[1: m], \\
\mathrm{a}_{i}^{\top} \mathrm{x}=b_{i} \mathrm{x}^{\top} \text { for } i \in[1: m]
\end{array}\right\}, \\
& \mathcal{L}_{3}=\left\{\left(\begin{array}{cc}
x_{0} \mathrm{x}^{\top} \\
\mathrm{x} & \mathrm{x}
\end{array}\right) \in \mathcal{S}^{n+1}: \begin{array}{l}
\mathrm{a}_{i}^{\top} \mathrm{x}=b_{i} x_{0} \text { for } i \in[1: m], \\
\mathrm{a}_{i}^{\top} \mathrm{X}_{i}=b_{i}^{2} x_{0} \quad \text { for } i \in[1: m]
\end{array}\right\}, \\
& \mathcal{L}_{4}=\left\{\left(\begin{array}{cc}
x_{0} \mathrm{x}^{\top} \\
\mathrm{x} & \mathrm{x}
\end{array}\right) \in \mathcal{S}^{n+1}: \sum_{i=1}^{m}\left(\mathrm{a}_{i}^{\top} \mathrm{X}_{i}-2 b_{i} \mathrm{a}_{i}^{\top} \mathrm{x}+b_{i}^{2} x_{0}\right)=0\right\} .
\end{aligned}
$$

Note that from the definition of $\mathrm{R}$, we have $\left(\mathrm{RYR}^{\top}\right)_{00}=(\mathrm{Y})_{00}$, and thus for $\mathcal{L}_{1}$, the additional constraint $x_{0}=1$ is equivalent to fixing $(\mathrm{Y})_{00}=1$.

In a similar vein, when dealing with binarity constraints, we will use the following linear subspaces:

$$
\begin{aligned}
& \mathcal{B}_{1}=\left\{\left(\begin{array}{cc}
x_{0} & \mathrm{x}^{\top} \\
\mathrm{x} & \mathrm{x}
\end{array}\right) \in \mathcal{S}^{n+1}: X_{j j}=x_{j} \quad \text { for } j \in B\right\}, \\
& \mathcal{B}_{2}=\left\{\left(\begin{array}{cc}
x_{0} & \mathrm{x}^{\top} \\
\mathrm{x} & \mathrm{x}
\end{array}\right) \in \mathcal{S}^{n+1}: \sum_{j \in B}\left(X_{j j}-x_{j}\right)=0\right\} .
\end{aligned}
$$

\section{Reformulation}

In this section we shall prove the equivalence of problems $(\overline{\mathrm{CPP}}),(7)$ and $(9)$ and similar results for the relaxations (8) and (10). We split this into several parts. We begin in Subsection 2.1 by considering sets $\mathcal{L}_{i}, i \in[1: 4]$. Then in Subsection 2.2 we consider sets $\mathcal{B}_{1}, \mathcal{B}_{2}$. In Subsection 2.3 we combine these to look at the optimization problems explicitly, and compare them to those that previously appeared in the literature.

\subsection{Aggregation of linear constraints}

In this subsection we will look at the four subspaces $\mathcal{L}_{i}$ related to the linear constraints, and we will now show that the intersections of $\mathcal{L}_{i}$ with the psd cone are the same for all $i \in[1: 4]$.

Theorem 1 We have

$$
\begin{aligned}
& \mathcal{L}_{1}=\mathcal{L}_{2} \subseteq \mathcal{L}_{3} \subseteq \mathcal{L}_{4}, \\
& \text { (a) } \mathcal{L}_{1} \cap \mathcal{S}_{+}^{n+1}=\mathcal{L}_{2} \cap \mathcal{S}_{+}^{n+1}=\mathcal{L}_{3} \cap \mathcal{S}_{+}^{n+1}=\mathcal{L}_{4} \cap \mathcal{S}_{+}^{n+1} \quad \text { and furthermore } \\
& \text { (c) } \mathcal{L}_{1} \cap \mathcal{S}_{+}^{n+1}=\mathcal{R S}_{+}^{n+1-m} \mathrm{R}^{\top} .
\end{aligned}
$$

Proof. From (4), applied to the columns of $\left(\begin{array}{cc}x_{0} & \mathrm{x}^{\top} \\ \mathrm{x} & \mathrm{x}\end{array}\right)=\mathrm{RYR}^{\top}$, it is easy to see that $\mathcal{L}_{1} \subseteq \mathcal{L}_{2}$ holds. The inclusions $\mathcal{L}_{2} \subseteq \mathcal{L}_{3} \subseteq \mathcal{L}_{4}$ are trivial. From (5) we have $\mathcal{L}_{1} \cap \mathcal{S}_{+}^{n+1}=\mathrm{RS}_{+}^{n+1-m} \mathrm{R}^{\top}$. We are thus left to show that $\mathcal{L}_{2} \subseteq \mathcal{L}_{1}$ for $(\mathrm{a})$, and $\mathcal{L}_{4} \cap \mathcal{S}_{+}^{n+1} \subseteq \mathcal{L}_{1}$ for (b).

First we shall show that $\mathcal{L}_{2} \subseteq \mathcal{L}_{1}$. The zero matrix is trivially in both of these sets and we now consider an arbitrary nonzero $Z=\left(\begin{array}{cc}x_{0} & \mathbf{X}^{\top} \\ \mathbf{x} & \mathbf{X}\end{array}\right) \in \mathcal{L}_{2}$. As $Z \in \mathcal{S}^{n+1}$, for $p=\operatorname{rank}(Z) \in[1: n+1]$, there exists $\{\boldsymbol{\zeta}, \boldsymbol{\mu}\} \subset \mathbb{R}^{p}$ and $\mathbf{z}_{1}, \ldots, \mathbf{z}_{p} \in \mathbb{R}^{n}$ such that $\mu_{k} \neq 0$ for all $k$ and $\left(\begin{array}{c}\zeta_{1} \\ \mathbf{z}_{1}\end{array}\right), \ldots,\left(\begin{array}{c}\zeta_{p} \\ \mathbf{z}_{p}\end{array}\right)$ are orthonormal and $\mathbf{Z}=\sum_{k=1}^{p} \mu_{k}\left(\begin{array}{l}\zeta_{k} \\ \mathbf{z}_{k}\end{array}\right)\left(\begin{array}{l}\zeta_{k} \\ \mathbf{z}_{k}\end{array}\right)^{\top}$. For all $i \in[1: m]$ and $l \in[1: p]$ we have

$$
0=\left(\begin{array}{c}
\zeta_{l} \\
\mathbf{z}_{l}
\end{array}\right)^{\top}\left(\begin{array}{l}
0 \\
0
\end{array}\right)=\left(\begin{array}{c}
\zeta_{l} \\
\mathrm{z}_{l}
\end{array}\right)^{\top}\left(\begin{array}{c}
\mathrm{a}_{i}^{\top} \mathrm{x}-b_{i} x_{0} \\
\mathrm{Xa}_{i}-b_{i} \mathrm{x}
\end{array}\right)=\left(\begin{array}{c}
\zeta_{l} \\
\mathrm{z}_{l}
\end{array}\right)^{\top} \mathrm{Z}\left(\begin{array}{c}
-b_{i} \\
\mathrm{a}_{i}
\end{array}\right)=\mu_{l}\left(\begin{array}{c}
\zeta_{l} \\
\mathrm{z}_{l}
\end{array}\right)^{\top}\left(\begin{array}{c}
-b_{i} \\
\mathrm{a}_{i}
\end{array}\right)=\mu_{l}\left(\mathrm{a}_{i}^{\top} \mathrm{z}_{l}-b_{i} \zeta_{l}\right) .
$$

By (4) this implies that for all $l \in[1: p]$ there exists $\mathrm{y}_{l} \in \mathbb{R}^{n+1-m}$ such that $\left(\begin{array}{l}\zeta_{l} \\ \mathrm{z}_{l}\end{array}\right)=\mathrm{Ry}_{l}$. Letting $\mathrm{Y}=$ $\sum_{k=1}^{p} \mu_{k} \mathrm{y}_{k} \mathrm{y}_{k}^{\top} \in \mathcal{S}^{n+1-m}$, we get $\mathrm{Z}=\mathrm{RYR}^{\top} \in \mathcal{L}_{1}$. 
We shall now show that $\mathcal{L}_{4} \cap \mathcal{S}_{+}^{n+1} \subseteq \mathcal{L}_{1}$ using a similar but different proof. We consider an arbitrary $\left(\begin{array}{cc}x_{0} & \mathbf{x}^{\top} \\ \mathbf{x} & \mathbf{x}\end{array}\right) \in \mathcal{L}_{4} \cap \mathcal{S}_{+}^{n+1}$. As $\left(\begin{array}{cc}x_{0} & \mathbf{x}^{\top} \\ \mathbf{x} & \mathbf{x}\end{array}\right) \in \mathcal{S}_{+}^{n+1}$, there exists $\left\{\boldsymbol{\zeta}, \mathbf{z}_{1}, \ldots, \mathbf{z}_{n}\right\} \subset \mathbb{R}^{n}$ such that $\left(\begin{array}{cc}x_{0} & \mathbf{x}^{\top} \\ \mathbf{x} & \mathbf{x}\end{array}\right)=\sum_{k=1}^{n}\left(\begin{array}{c}\zeta_{k} \\ \mathbf{z}_{k}\end{array}\right)\left(\begin{array}{c}\zeta_{k} \\ \mathbf{z}_{k}\end{array}\right)^{\top}$. We then have $0=\sum_{i=1}^{m}\left(\mathbf{a}_{i}^{\top} \mathbf{X a}_{i}-2 b_{i} \mathbf{a}_{i}^{\top} \mathbf{x}+b_{i}^{2} x_{0}\right)=\sum_{i=1}^{m} \sum_{k=1}^{n}\left(\mathbf{a}_{i}^{\top} \mathbf{z}_{k}-b_{i} \zeta_{k}\right)^{2} \geq 0$.

Therefore $\mathrm{a}_{i}^{\top} \mathrm{z}_{k}=b_{i} \zeta_{k}$ for all $i, k$, and thus, from (4), for all $k$ there exists $\mathrm{y}_{k} \in \mathbb{R}^{n+1-m}$ such that $\left(\begin{array}{c}\zeta_{k} \\ \mathrm{z}_{k}\end{array}\right)=$ $\mathrm{Ry}_{k}$. Now letting $\mathrm{Y}=\sum_{k=1}^{n} \mathrm{y}_{k} \mathrm{y}_{k}^{\top} \in \mathcal{S}^{n+1-m}$, we get $\mathrm{Z}=\mathrm{RYR}^{\top} \in \mathcal{L}_{1}$.

In spite of these equivalences, we still distinguish the different spaces $\mathcal{L}_{i}$, even though their intersections with the psd cone coincide. The difference in descriptions is especially important when considering optimization problems and their duals later in the paper. Recall that dual problems significantly depend on the primal formulation or description of the same problem.

\subsection{Aggregation of binary constraints}

In this subsection we will consider the subspaces $\mathcal{B}_{1}$ and $\mathcal{B}_{2}$ related to the binary constraints of $(\mathrm{P})$. Similarly to the previous subsection, we will see that when intersected with certain sets, the resulting intersection is independent of the choice of $\mathcal{B}_{1}$ or $\mathcal{B}_{2}$. To show this we first need the following two results:

Lemma 1 Let $j \in[1: n]$ and assume that there is a $\mu \in \mathbb{R}$ such that $x_{j} \leq \mu$ for all $\mathrm{x} \in \mathcal{Z}$. Then for $\left(\begin{array}{c}x_{0} \mathrm{x}^{\top} \\ \mathrm{x}\end{array}\right) \in$ $\mathcal{L}_{2} \cap \mathcal{N}^{n+1}$ we have $X_{j k} \leq \mu x_{k}$ for all $k \in[1: n]$.

Proof. Consider an arbitrary $k \in[1: n]$ and let $\mathbf{Z}=\left(\begin{array}{cc}x_{0} & \mathrm{x}^{\top} \\ \mathbf{x} & \mathrm{X}\end{array}\right)$. From $\mathbf{Z} \in \mathcal{N}^{n+1}$ we have $x_{k} \geq 0$ and we now consider two cases:

1. If $x_{k}=0$ then let $\widehat{x}$ be the $k^{\text {th }}$ column of $X$. From $Z \in \mathcal{N}^{n+1}$ we have $\widehat{x} \in \mathbb{R}_{+}^{n}$, and from $Z \in \mathcal{L}_{2}$, for all $i \in[1: m]$ we have $\mathrm{a}_{i}^{\top} \widehat{\mathrm{x}}=\left(\mathrm{a}_{i}^{\top} \mathrm{X}\right)_{k}=\left(b_{i} \mathrm{x}^{\top}\right)_{k}=b_{i} x_{k}=0$. Therefore $\widehat{\mathrm{x}} \in \mathcal{Z}_{\infty}$ and by looking at $\mathrm{v}=\mathrm{x}_{0}+t \widehat{\mathrm{x}}$ with $t \uparrow \infty$, this implies that $0=\widehat{x}_{j}=X_{j k}$.

2. If $x_{k}>0$ then let $\widehat{x}$ be the $k^{\text {th }}$ column of $\frac{1}{x_{k}} X$. From $Z \in \mathcal{N}^{n+1}$ we have $\widehat{x} \in \mathbb{R}_{+}^{n}$, and from $Z \in \mathcal{L}_{2}$, for all $i \in[1: m]$ we have $\mathrm{a}_{i}^{\top} \widehat{\mathrm{x}}=\frac{1}{x_{k}}\left(\mathrm{a}_{i}^{\top} \mathrm{X}\right)_{k}=\frac{1}{x_{k}}\left(b_{i} \mathrm{x}^{\boldsymbol{x}_{k}}\right)_{k}=b_{i}$. Therefore $\widehat{\mathrm{x}} \in \mathcal{Z}$ and thus $\mu \geq \widehat{x}_{j}=\frac{1}{x_{k}} X_{j k}$.

Lemma 2 We have $\mathcal{B}_{1} \cap \mathcal{L}_{2} \cap \mathcal{N}^{n+1}=\mathcal{B}_{2} \cap \mathcal{L}_{2} \cap \mathcal{N}^{n+1}$

Proof. We trivially have $\mathcal{B}_{1} \subseteq \mathcal{B}_{2}$, and the proof will be completed if we can show $\mathcal{B}_{2} \cap \mathcal{L}_{2} \cap \mathcal{N}^{n+1} \subseteq \mathcal{B}_{1}$. Considering an arbitrary $\left(\begin{array}{cc}x_{0} & \mathrm{x}^{\top} \\ \mathrm{x} & \mathrm{x}\end{array}\right) \in \mathcal{B}_{2} \cap \mathcal{L}_{2} \cap \mathcal{N}^{n+1}$, from Lemma $]$ and $(2)$, for all $j \in B$ we have $X_{j j}-x_{j} \leq 0$ and thus $0=\sum_{k \in B}\left(X_{k k}-x_{k}\right) \leq X_{j j}-x_{j} \leq 0$. Therefore $\left(\begin{array}{cc}x_{0} & \mathbf{x}^{\top} \\ \mathbf{x} & \mathrm{x}\end{array}\right) \in \mathcal{B}_{1}$.

We are now ready to present a key result in establishing equivalence of formulations for binarity constraints:

Proposition 1 For all $i, j \in[1: 4]$ and $k, l \in[1: 2]$ we have

$$
\begin{aligned}
\mathcal{B}_{1} & \subseteq \mathcal{B}_{2} \\
\mathcal{B}_{k} \cap \mathcal{L}_{i} \cap \mathcal{C} \mathcal{P}^{n+1} & =\mathcal{B}_{l} \cap \mathcal{L}_{j} \cap \mathcal{C P}^{n+1}, \\
\mathcal{B}_{k} \cap \mathcal{L}_{i} \cap \mathcal{S}_{+}^{n+1} \cap \mathcal{N}^{n+1} & =\mathcal{B}_{l} \cap \mathcal{L}_{j} \cap \mathcal{S}_{+}^{n+1} \cap \mathcal{N}^{n+1} .
\end{aligned}
$$

Proof. We trivially have $\mathcal{B}_{1} \subseteq \mathcal{B}_{2}$. Noting that $\mathcal{C} \mathcal{P}^{n+1} \subseteq \mathcal{S}_{+}^{n+1} \cap \mathcal{N}^{n+1}$ and using Theorem 1 , without loss of generality we assume $i=j=2$. The results then follow directly from Lemma 2 . 


\subsection{Optimization problems: New CP formulations and relaxations}

For $i \in[1: 4]$ and $k \in[1: 2]$ we will now consider the problem

$$
\min \left\{\langle\mathrm{Q}, \mathrm{X}\rangle+2 \mathrm{c}^{\top} \mathrm{x}:\left(\begin{array}{cc}
1 & \mathrm{x}^{\top} \\
\mathrm{x} & \mathrm{X}
\end{array}\right) \in \mathcal{B}_{k} \cap \mathcal{L}_{i} \cap \mathcal{C} \mathcal{P}^{n+1}\right\} .
$$

Theorem 2 All problems (11) are equivalent for any choice of $i \in[1: 4]$ and $k \in[1: 2]$. In particular, this includes problem [CPP, which was shown in [10] by Burer to have the same optimal value as $(\mathrm{P})$.

Proof. For $(i, k)=(3,1)$, problem (11) expressed explicitly is in fact problem CPP. From Proposition 1 and the results of [10, we therefore deduce that they all have the same optimal value as $(\mathrm{P})$. In particular, considering (11) expressed explicitly for $(i, k)=(4,2)$ and $(i, k)=(1,2)$ we get problems (7) and (9) respectively.

In [10, Burer showed this result for problem (11) with $i=3, k=1$. This is then a completely positive problem $\mathrm{CPP}$ with a completely positive constraint of order $n+1$ and $2 m+|B|$ linear equality constraints.

If we alternatively aggregate the constraints by considering problem (11) with $i=4, k=2$ we get the equivalent completely positive problem (7), which has only two homogeneous linear equality constraints and a completely positive constraint of order $n+1$.

In [1], the authors introduced an equivalent copositive problem with only one equality constraint, however in general this can have a larger order completely positive constraint, possibly even as high as order $2 n+1$.

Unfortunately, optimizing over the completely positive cone is a very difficult problem (in fact an NP-hard problem [6, 15, 18]), and thus we wish to consider approximations of this. A natural relaxation of problem (11) is

$$
\min \left\{\langle\mathrm{Q}, \mathrm{X}\rangle+2 \mathrm{c}^{\top} \mathrm{x}:\left(\begin{array}{cc}
1 & \mathrm{x}^{\top} \\
\mathrm{x} & \mathrm{X}
\end{array}\right) \in \mathcal{B}_{k} \cap \mathcal{L}_{i} \cap \mathcal{S}_{+}^{n+1} \cap \mathcal{N}^{n+1}\right\} .
$$

Theorem 3 All problems (12) are equivalent for any choice of $i \in[1: 4]$ and $k \in[1: 2]$. In particular, for $(i, k)=$ $(3,1)$, we obtain the following natural relaxation to $\mathrm{CPP}$ :

$$
\begin{aligned}
\min & \langle\mathrm{Q}, \mathrm{X}\rangle+2 \mathrm{c}^{\top} \mathrm{x} \\
\text { s.t. } & \mathrm{a}_{i}^{\top} \mathrm{x}=b_{i} \text { for } i \in[1: m] \\
& \left\langle\mathrm{a}_{i} \mathrm{a}_{i}^{\top}, \mathrm{X}\right\rangle=b_{i}^{2} \quad \text { for } i \in[1: m] \\
& X_{j j}=x_{j} \text { for all } j \in B \\
& \left(\begin{array}{l}
1 \\
\mathrm{x} \\
\mathrm{x}
\end{array}\right) \in \mathcal{S}_{+}^{n+1} \cap \mathcal{N}^{n+1} .
\end{aligned}
$$

while for the choice $(i, k)=(1,1)$ we arrive at

$$
\begin{array}{ll}
\min & \langle\mathrm{Q}, \mathrm{X}\rangle+2 \mathrm{c}^{\top} \mathrm{x} \\
\text { s.t. } & X_{j j}=x_{j} \text { for all } j \in B \\
& \mathrm{RYR}^{\top}=\left(\begin{array}{ll}
1 & \mathrm{x}^{\top} \\
\mathrm{x} & \mathrm{X}
\end{array}\right) \\
& \left(\begin{array}{cc}
1 & \mathrm{x}^{\top} \\
\mathrm{x} & \mathrm{X}
\end{array}\right) \in \mathcal{N}^{n+1}, \mathrm{Y} \in \mathcal{S}_{+}^{n+1-m} .
\end{array}
$$

and for the choice of $(i, k)=(1,2)$ the explicit expression was already given in the introduction as problem (10).

Proof. Again from Proposition 1 we have that problems 12 are equivalent for all choices of $i$ and $k$. The remaining claims are straightforward.

Problem 130 is the natural relaxation of Burer's completely positive formulation from [10, and is an SDP with $2 m+|B|$ linear equality constraints, $\frac{1}{2} n(n-1)-|B|$ inequality constraints and a psd constraint of order $n+1$. Indeed, we have $\frac{1}{2} n(n-1)$ inequality constraints for $\mathcal{N}^{n}$, rather than the expected $\frac{1}{2} n(n+1)$ inequality constraints as the on-diagonal entries are already restricted to be nonnegative by the psd constraint; the same reasoning, using $x_{j}=X_{j j}$ applies to all $j \in B$, reducing the number of inequality constraints further. Computationally, 
this problem is still fairly difficult, mainly due to the fact that we have large psd constraints and we never have an interior point for the problem. Indeed, for any feasible $(1, \mathrm{x}, \mathrm{X})$ and any $i$ we have

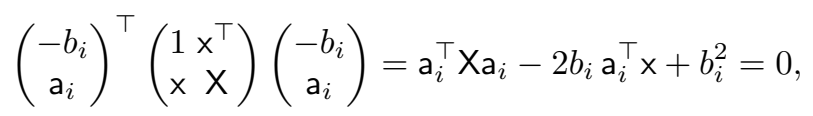

which implies that $\left(\begin{array}{ll}1 & x^{\top} \\ x & x\end{array}\right)$ is on the boundary of the psd cone.

In [1], Burer showed how we can deal with the linear constraints to give the relaxation (14), which solves part of these difficulties: if there are no binary constraints, then for $\varepsilon>0$ small enough the point $Y=\varepsilon l+(1-\varepsilon) \mathrm{e}_{0} \mathrm{e}_{0}^{\top}$ is strictly feasible for this problem. If there are binary variables then unfortunately we are unable to guarantee strict feasibility (or even feasibility). This relaxation is an SDP with $|B|$ linear equality constraints, $\frac{1}{2} n(n-1)-|B|$ inequality constraints and a psd constraint of order $n+1-m$. This means the psd constraint is of smaller order than before, and whilst this advantage is less pronounced in case $m \ll n$, it is still of significance in view of the fact that handling psd constraints (LMIs) requires in general more effort than handling linear constraints. The formulation (14) still has the problem that if $|B|$ is large then we have a large number of linearized binary constraints. This can be avoided by considering the equivalent problem 10, , which is an SDP with one linear equality constraint, $\frac{1}{2} n(n-1)-|B|$ inequality constraints and a psd constraint of order $n+1-m$. Further support for this choice will be provided when we consider the dual problems in Subsection 3.2. Note that the number of nonnegativity constraints (membership of $\left(\begin{array}{ll}1 & x^{\top} \\ x & x\end{array}\right) \in \mathcal{N}^{n+1}$ ) is the same in all formulations, so again this advantage is less pronounced if $|B| \ll n$.

\section{Tightening duality gaps}

We will now investigate the dual problems to the reformulations introduced in the first half of this paper. In particular we will look at how the duality gap is affected by the choice of reformulation. We will also consider when we can guarantee there is a zero duality gap through Slater's condition holding in the dual.

\subsection{Conic optimization: role of closure in duality gaps}

We begin by recalling basic results on a more general class of conic optimization problems. For ease of reference the proofs of these results will be provided here although some may be found scattered in the literature.

We will primarily consider conic optimization problems of the following form:

$$
\begin{array}{cl}
\min _{x} & \langle\mathrm{q}, \mathrm{x}\rangle \\
\text { s.t. } & \left\langle\mathrm{d}_{0}, \mathrm{x}\right\rangle=1, \\
& x \in \mathcal{L} \cap \mathcal{K},
\end{array}
$$

where $\mathcal{K} \subseteq \mathbb{R}^{n}$ is a closed convex pointed cone and $\mathcal{L}=\left\{\mathrm{d}_{1}, \ldots, \mathrm{d}_{m}\right\}^{\perp}=\left\{\mathrm{x} \in \mathbb{R}^{n}:\left\langle\mathrm{d}_{i}, \mathrm{x}\right\rangle=0\right.$, for $\left.i \in[1: m]\right\}$ is a linear subspace. We further assume that

$$
\left\{\mathrm{q}, \mathrm{d}_{0}, \mathrm{~d}_{1}, \ldots, \mathrm{d}_{m}\right\} \subset \mathbb{R}^{n} \text { are such that }\left\langle\mathrm{d}_{0}, \mathrm{x}\right\rangle \geq 0 \text { for all } \mathrm{x} \in \mathcal{L} \cap \mathcal{K} \text {, i.e. } \mathrm{d}_{0} \in(\mathcal{L} \cap \mathcal{K})^{*} .
$$

Formulation (15) looks special at first glance but can be as well applied to general conic optimization problems as detailed in Theorem 5 below.

In order to consider the Lagrangian dual problem to 15 , we could consider $\mathcal{L} \cap \mathcal{K}$ to be a single closed convex cone and use the Lagrangian function $\bar{\Lambda}: \mathbb{R}^{n} \times \mathbb{R} \times(\mathcal{L} \cap \mathcal{K})^{*} \rightarrow \mathbb{R}$, given by

$$
\bar{\Lambda}(\mathrm{x} ; \mathrm{y}, \mathrm{z})=\langle\mathrm{q}, \mathrm{x}\rangle+y\left(1-\left\langle\mathrm{d}_{0}, \mathrm{x}\right\rangle\right)-\langle\mathrm{z}, \mathrm{x}\rangle=y+\left\langle\mathrm{q}-y \mathrm{~d}_{0}-\mathrm{z}, \mathrm{x}\right\rangle .
$$

Letting opt 15 be the optimal value to problem $(15)$, we get

$$
\operatorname{opt} 15]=\min _{\mathbf{x} \in \mathbb{R}^{n}} \max _{\substack{y \in \mathbb{R}, \mathbf{z} \in(\mathcal{L} \cap \mathcal{K})^{*}}} \bar{\Lambda}(\mathrm{x} ; y, \mathrm{z}) \geq \max _{\substack{y \in \mathbb{R}, \mathbf{z} \in(\mathcal{L} \cap \mathcal{K})^{*}}} \min _{\mathbf{x} \in \mathbb{R}^{n}} \bar{\Lambda}(\mathrm{x} ; y, \mathrm{z})=\max _{\substack{y \in \mathbb{R}, \mathrm{z} \in(\mathcal{L} \cap \mathcal{K})^{*}}}\left\{y: \mathrm{q}-y \mathrm{~d}_{0}=\mathrm{z}\right\}
$$


and thus the dual problem is

$$
\begin{array}{cl}
\max _{y} & y \\
\text { s.t. } & \mathbf{q}-y \mathrm{~d}_{0} \in(\mathcal{L} \cap \mathcal{K})^{*} .
\end{array}
$$

This approach has the advantage of zero duality gap even if Slater's condition fails on both sides:

Theorem 4 Provided at least one of problems (15) and (17) are feasible, we have opt (15) = opt (17) even if Slater's condition is violated for both problems.

Proof. We have that opt 15$] \geq$ opt 17 and thus if opt $17=\infty$ then the result is trivial. From now on we suppose that opt 17$)<\infty$ and consider an arbitrary $\lambda>$ opt $(17)$. We shall show that $\lambda>$ opt (15), and thus opt 15 opt 17 ), completing the proof.

As $\lambda>$ opt (17) we have $\mathrm{q}-\lambda \mathrm{d}_{0} \notin(\mathcal{L} \cap \mathcal{K})^{*}$, and thus there exists $\mathrm{z} \in \mathcal{L} \cap \mathcal{K}$ such that $\left\langle\mathrm{z}, \mathrm{q}-\lambda \mathrm{d}_{0}\right\rangle\langle 0$ and from the assumption on $\mathrm{d}_{0}$ we have $\left\langle\mathrm{z}, \mathrm{d}_{0}\right\rangle \geq 0$. We now consider two cases:

1. If $\left\langle z, \mathrm{~d}_{0}\right\rangle=0$ then $\left\langle\mathrm{z}, \mathrm{q}-y \mathrm{~d}_{0}\right\rangle=\langle\mathrm{z}, \mathrm{q}\rangle=\left\langle\mathrm{z}, \mathrm{q}-\lambda \mathrm{d}_{0}\right\rangle\langle 0$ for all $y \in \mathbb{R}$ and thus (17) is infeasible. As we assume at least one of the problems is feasible, there exists an $x$ which is feasible for (15). Therefore for all $\mu \geq 0$ we have that $x+\mu z$ is feasible for $(15)$, and considering $\mu \rightarrow \infty$ we get opt $15 p-\infty<\lambda$.

2. If $\left\langle z, d_{0}\right\rangle>0$ then without loss of generality $\left\langle z, d_{0}\right\rangle=1$. We then have that $z$ is feasible for 15 and $\lambda>\left\langle\mathrm{z}, \mathrm{q}-\lambda \mathrm{d}_{0}\right\rangle+\lambda=\langle\mathrm{z}, \mathrm{q}\rangle \geq \operatorname{opt}(15)$.

The main disadvantage of this approach is that we often miss a practical description of the dual feasible set $(\mathcal{L} \cap \mathcal{K})^{*}$. Although we have $(\mathcal{L} \cap \mathcal{K})^{*}=\operatorname{cl}\left(\mathcal{L}^{\perp}+\mathcal{K}^{*}\right)$, characterizing this closure is a nontrivial problem, and hence we are unable to use this dual in practice. Instead we may consider separating the conic and linear constraints, and then taking the resulting (possibly different) Lagrangian dual. By this we mean first noting that

$$
\operatorname{opt} 15=\min _{\mathrm{u}, \mathrm{x}}\left\{\langle\mathrm{q}, \mathrm{x}\rangle: 1=\left\langle\mathrm{d}_{0}, \mathrm{x}\right\rangle, \mathrm{x} \in \mathcal{L}, \mathrm{x} \in \mathcal{K}\right\}
$$

Then considering the corresponding Lagrangian function, $\Lambda: \mathbb{R}^{n} \times \mathbb{R} \times \mathcal{L}^{\perp} \times \mathcal{K}^{*} \rightarrow \mathbb{R}$, given by

$$
\Lambda(\mathrm{x} ; \mathrm{y}, \mathrm{w}, \mathrm{z})=\langle\mathrm{q}, \mathrm{x}\rangle+y\left(1-\left\langle\mathrm{d}_{0}, \mathrm{x}\right\rangle\right)-\langle\mathrm{w}, \mathrm{x}\rangle-\langle\mathrm{z}, \mathrm{x}\rangle=y+\left\langle\mathrm{x}, \mathrm{q}-y \mathrm{~d}_{0}-\mathrm{w}-\mathrm{z}\right\rangle,
$$

we get

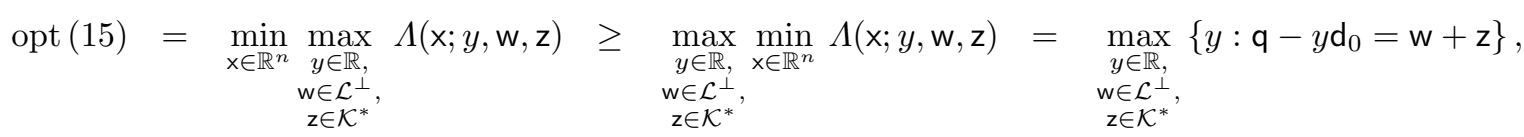

and thus the dual problem is

$$
\begin{array}{ll}
\max _{y} & y \\
\text { s.t. } & \mathbf{q}-y \mathrm{~d}_{0} \in \mathcal{L}^{\perp}+\mathcal{K}^{*} .
\end{array}
$$

This dual problem could have alternatively been obtained as a modification of our original dual problem (17) by ignoring the closure operation in $(\mathcal{L} \cap \mathcal{K})^{*}=\operatorname{cl}\left(\mathcal{L}^{\perp}+\mathcal{K}^{*}\right)$. Thereby, the feasible set of the dual shrinks and the duality gap may increase.

Example 1 ([9, Example 3.3]) Consider problem (15) and its duals [17) and (18) for

$$
\mathrm{Q}=\left(\begin{array}{ccc}
0 & 0 & 0 \\
0 & 0 & 0 \\
0 & 0 & 1
\end{array}\right), \quad \mathrm{D}_{0}=\left(\begin{array}{lll}
0 & 1 & 0 \\
1 & 0 & 0 \\
0 & 0 & 1
\end{array}\right), \quad \mathcal{L}=\left\{\mathrm{X} \in \mathcal{S}^{3}: x_{11}=0\right\}, \quad \mathcal{K}=\mathcal{C P}^{3}
$$

Considering the primal problem, we have

$$
\begin{gathered}
\qquad \mathcal{L} \cap \mathcal{K}=\left\{\mathrm{X} \in \mathcal{C P}^{3}: x_{11}=0\right\}=\left\{\mathrm{X} \in \mathcal{S}^{3}: x_{1 i}=0 \text { for } i \in[1: 3],\left(\begin{array}{l}
x_{22} x_{23} \\
x_{23} x_{33}
\end{array}\right) \in \mathcal{C} \mathcal{P}^{2}\right\}, \\
\text { feas } 15]=\left\{\mathrm{X} \in \mathcal{S}^{3}: x_{1 i}=0 \text { for } i \in[1: 3], x_{33}=1, x_{23} \geq 0, x_{23}^{2} \leq x_{22}\right\}, \quad \text { opt } 15=1 .
\end{gathered}
$$


For the dual problems, from basic results on copositivity (see e.g. [14, Theorem 1.1]) we have

$$
\mathcal{N}^{3} \subseteq \mathcal{L}^{\perp}+\mathcal{K}^{*}=\left\{\lambda\left(\begin{array}{lll}
1 & 0 & 0 \\
0 & 0 & 0 \\
0 & 0 & 0
\end{array}\right): \lambda \in \mathbb{R}\right\}+\mathcal{C O O P} \mathcal{P}^{3} \subseteq\left\{\begin{array}{c}
\left.\mathrm{Z} \in \mathcal{S}^{3}: \begin{array}{c}
z_{22} z_{23} \\
z_{23} z_{33}
\end{array}\right) \in \mathcal{C O O} \mathcal{P}^{2} \\
z_{22}=0 \Rightarrow z_{12} \geq 0
\end{array}\right\}
$$

and thus feas $(18)=\{y \in \mathbb{R}: y \leq 0\}$ and opt $180=0<$ opt 115 . Note that $N^{3} \subset \mathcal{C O} \mathcal{P}^{3}$.

Considering an arbitrary $\mathrm{Z} \in \mathcal{S}^{3}$ with $\left(\begin{array}{ll}z_{22} & z_{23} \\ z_{23} & z_{33}\end{array}\right) \in \mathcal{C O} \mathcal{P}^{2}$, from [5, Lemma 4.1] we get that $\mathrm{Z}+\varepsilon \mathbf{l}_{3} \in \mathcal{L}^{\perp}+\mathcal{K}^{*}$ for all $\varepsilon>0$. Combining this observation with the inclusion relations above, we get

$$
\operatorname{cl}\left(\mathcal{L}^{\perp}+\mathcal{K}^{*}\right)=\left\{\mathrm{Z} \in \mathcal{S}^{3}:\left(\begin{array}{ll}
z_{22} & z_{23} \\
z_{23} & z_{33}
\end{array}\right) \in \mathcal{C O} \mathcal{P}^{2}\right\}=(\mathcal{L} \cap \mathcal{K})^{*},
$$

and thus feas 17$)=\{y \in \mathbb{R}: y \leq 1\}$ and opt 17$)=1=$ opt 15 .

However, the advantage of this approach is that we have $\mathcal{L}^{\perp}=\operatorname{span}\left\{\mathrm{d}_{i}: i \in[1: m]\right\}$ and we generally know a characterization of the set $\mathcal{K}^{*}$. Due to this advantage it shall be this dual that we consider from now on in the paper.

But before let us address the general case. The following theorem applies the results so far presented in this subsection to general conic optimization problems.

Theorem 5 In general conic optimization, all nontrivial dual gaps (i.e., when at least one of the primal or dual problems is feasible) are caused by ignoring the closure operation. To be more precise, consider the following general conic optimization problem:

$$
\begin{array}{ll}
\min _{x} & \langle\widetilde{\mathbf{q}}, \mathbf{z}\rangle \\
\text { s.t. } & \left\langle\widetilde{\mathrm{d}}_{i}, \mathrm{z}\right\rangle=f_{i} \quad \text { for } i \in[1: m], \\
& \mathbf{z} \in \mathcal{C},
\end{array}
$$

where $\left\{\widetilde{\mathrm{q}}, \widetilde{\mathrm{d}}_{1}, \ldots, \widetilde{\mathrm{d}}_{m}\right\} \subset \mathbb{R}^{n-1}$, and $\mathcal{C} \subseteq \mathbb{R}^{n-1}$ is a closed convex pointed cone. Then the standard dual is given by

$$
\begin{array}{ll}
\max _{\mathrm{y}} & \mathrm{f}^{\top} \mathrm{y} \\
\text { s.t. } & \widetilde{\mathrm{q}}-\sum_{i=1}^{m} y_{i} \widetilde{\mathrm{d}}_{i} \in \mathcal{C}^{*},
\end{array}
$$

and this problem emerges by removing the closure operation as in transition from (17) to (18).

Proof. Problem $\sqrt{19})$ is equivalent to problem $(15)$ with $\mathcal{K}=\mathcal{C} \times \mathbb{R}_{+} \subseteq \mathbb{R}^{n-1} \times \mathbb{R}, \mathrm{q}=(\widetilde{\mathrm{q}}, 0) \in \mathbb{R}^{n-1} \times \mathbb{R}$, $\mathrm{d}_{0}=(\mathrm{o}, 1) \in \mathbb{R}^{n-1} \times \mathbb{R}$ and $\mathrm{d}_{i}=\left(\widetilde{\mathrm{d}}_{i},-f_{i}\right) \in \mathbb{R}^{n-1} \times \mathbb{R}$ for $i \in[1: m]$. The dual problem of the form (17) for this is then

$$
\begin{array}{ll}
\max _{y_{0}} & y_{0} \\
\text { s.t. } & \left(\widetilde{\mathrm{q}},-y_{0}\right) \in \operatorname{cl}\left(\left(\mathcal{C}^{*} \times \mathbb{R}_{+}\right)+\operatorname{span}\left\{\left(\widetilde{\mathrm{d}}_{i},-f_{i}\right): i \in[1: m]\right\}\right) .
\end{array}
$$

Removing the closure to give problem [18), and then simplifying, gives the problem 20].

In order to guarantee equality of the optimal values we will use Slater's condition. This requires the existence of a so called strictly feasible point, and we know from the discussion in Subsection 2.3 that in general our primal problems will not contain such a point. For this reason we will focus on the dual side. We will use the following well known theorem. For the readers' convenience, we include a proof in the appendix.

Theorem 6 Consider problems 15] and (18). Under the assumptions 16) we have

$$
\operatorname{int}\left(\mathcal{L}^{\perp}+\mathcal{K}^{*}\right)=\mathcal{L}^{\perp}+\operatorname{int}\left(\mathcal{K}^{*}\right) \neq \emptyset,
$$

and we say that Slater's condition holds for 18 if 
1. there exists $\widehat{y} \in \mathbb{R}$ such that $\mathrm{q}-\widehat{y} \mathrm{~d}_{0} \in \operatorname{int}\left(\mathcal{L}^{\perp}+\mathcal{K}^{*}\right)$.

An equivalent condition to this is:

2. $\langle\mathrm{q}, \mathrm{u}\rangle>0$ for all $\mathrm{u} \in \mathcal{L} \cap \mathcal{K} \backslash\{\mathrm{o}\}$ such that $\left\langle\mathrm{d}_{0}, \mathrm{u}\right\rangle=0$.

If Slater's condition holds for $(18)$ then we have opt $\sqrt{15})=$ opt $(18)$.

If feas 150 then another two equivalent conditions to Slater's condition for (18) are:

3. there exists $\mu \in \mathbb{R} \cup\{\infty\}$ such that the set $\{\mathrm{x} \in$ feas 15$):\langle\mathrm{q}, \mathrm{x}\rangle \leq \mu\}$ is nonempty and compact,

4. for all $\mu \in \mathbb{R}$ we have that the set $\{\mathrm{x} \in$ feas $[15):\langle\mathrm{q}, \mathrm{x}\rangle \leq \mu\}$ is compact.

Therefore, if feas $[15 \neq \emptyset$ and Slater's condition holds for $\sqrt{18}$ then the optimal value to (15) is attained.

Corollary 1 If feas (15) is nonempty and bounded then Slater's condition holds for (18).

Remark 1 Combining Theorem 6 with Theorem 5, we have that Slater's condition holding for the dual problem (i.e., problem 21] with the closure removed) is equivalent to the following:

1. there exists $\mathrm{y} \in \mathbb{R}^{m}$ such that $\widetilde{\mathrm{q}}-\sum_{i=1}^{m} y_{i} \widetilde{\mathrm{d}}_{i} \in \operatorname{int} \mathcal{C}^{*}$,

2. $\langle\widetilde{\mathrm{q}}, \mathrm{z}\rangle>0$ for all $\mathrm{z} \in \mathcal{C} \backslash\{\mathrm{o}\}$ such that $\left\langle\widetilde{\mathrm{d}}_{i}, \mathrm{z}\right\rangle=0$ for $i \in[1: m]$.

When feas $[19] \neq \emptyset$ then a further two equivalent conditions are:

3. there exists $\mu \in \mathbb{R} \cup\{\infty\}$ such that the set $\{z \in$ feas $[19):\langle\widetilde{\mathbf{q}}, \mathrm{z}\rangle \leq \mu\}$ is nonempty and compact,

4. for all $\mu \in \mathbb{R}$ the set $\{z \in$ feas $(19):\langle\widetilde{\mathrm{q}}, z\rangle \leq \mu\}$ is compact.

\subsection{Our problems}

All of the results of the previous subsection naturally extend to other Euclidean spaces, including the spaces $\mathbb{R} \times \mathbb{R}^{n} \times \mathcal{S}^{n}$ and $\mathcal{S}^{n+1-m}$ which shall be considered in this subsection.

For $i \in[1: 4]$ and $k \in[1: 2]$, we will consider the dual problems to

$$
\begin{array}{ll}
\min & \langle\mathrm{Q}, \mathrm{X}\rangle+2 \mathrm{c}^{\top} \mathrm{x} \\
\text { s.t. } & x_{0}=1 \\
& \left(\begin{array}{cc}
x_{0} & \mathrm{x}^{\top} \\
\mathrm{x} & \mathrm{X}
\end{array}\right) \in \mathcal{B}_{k} \cap \mathcal{L}_{i} \cap \mathcal{C} \mathcal{P}^{n+1},
\end{array}
$$

and the duals of their relaxations

$$
\begin{array}{ll}
\min & \langle\mathrm{Q}, \mathrm{X}\rangle+2 \mathrm{c}^{\top} \mathrm{x} \\
\text { s.t. } & x_{0}=1 \\
& \left(\begin{array}{cc}
x_{0} & \mathrm{x}^{\top} \\
\mathrm{x} & \mathrm{X}
\end{array}\right) \in \mathcal{B}_{k} \cap \mathcal{L}_{i} \cap \mathcal{S}_{+}^{n+1} \cap \mathcal{N}^{n+1} .
\end{array}
$$

For $i=1$ this relaxation is equivalent to the following reduced problem, which we will also consider the dual of:

$$
\begin{array}{ll}
\min _{\mathrm{Y}} & \left\langle\mathrm{R}^{\top}\left(\begin{array}{ll}
0 & \mathrm{C}^{\top} \\
\mathrm{c} & \mathrm{Q}
\end{array}\right) \mathrm{R}, \mathrm{Y}\right\rangle \\
\text { s.t. } & \left\langle\mathrm{R}^{\top}\left(\begin{array}{ll}
1 & \mathrm{o}^{\top} \\
0 & 0
\end{array}\right) \mathrm{R}, \mathrm{Y}\right\rangle=1 \\
& \mathrm{RYR}^{\top} \in \mathcal{B}_{k} \\
& \mathrm{RYR}^{\top} \in \mathcal{N}^{n+1} \\
& \mathrm{Y} \in \mathcal{S}_{+}^{n+1-m} .
\end{array}
$$


We begin by considering problems $(22)$ and $(23)$ (for $k \in[1: 2]$ and $i \in[1: 4]$ ). In order to apply the results from the previous subsection we note that $\mathcal{B}_{k} \cap \mathcal{L}_{i}$ is a linear subspace with $\left(\mathcal{B}_{k} \cap \mathcal{L}_{i}\right)^{\perp}=\mathcal{B}_{k}^{\perp}+\mathcal{L}_{i}^{\perp}$, where

$$
\begin{aligned}
& \mathcal{L}_{1}^{\perp}=\left\{\left(\begin{array}{cc}
x_{0} & \mathrm{x}^{\top} \\
\mathrm{x} & \mathrm{x}
\end{array}\right) \in \mathcal{S}^{n+1}: \mathrm{R}^{T}\left(\begin{array}{cc}
x_{0} & \mathrm{x}^{\top} \\
\mathrm{x} & \mathrm{x}
\end{array}\right) \mathrm{R}=\mathrm{O}\right\}, \\
& \mathcal{L}_{2}^{\perp}=\operatorname{span}\left\{\left(\begin{array}{cc}
2 b_{i} & -\mathrm{a}_{i}^{\top} \\
-\mathrm{a}_{i} & \mathrm{O}
\end{array}\right): i \in[1: m]\right\}+\operatorname{span}\left\{\left(\begin{array}{cc}
0 & -b_{i} \mathrm{e}_{j}^{\top} \\
-b_{i} \mathrm{e}_{j} \mathrm{a}_{i} \mathrm{e}_{j}^{\top}+\mathrm{e}_{j} \mathrm{a}_{i}^{\top}
\end{array}\right): i \in[1: m], j \in[1: n]\right\}, \\
& \mathcal{L}_{3}^{\perp}=\operatorname{span}\left\{\left(\begin{array}{cc}
2 b_{i} & -\mathrm{a}_{i}^{\top} \\
-\mathrm{a}_{i} & \mathrm{O}
\end{array}\right): i \in[1: m]\right\}+\operatorname{span}\left\{\left(\begin{array}{cc}
b_{i}^{2} & \mathrm{o}^{\top} \\
0 & -\mathrm{a}_{i} \mathrm{a}_{i}^{\top}
\end{array}\right): i \in[1: m]\right\}, \\
& \mathcal{L}_{4}^{\perp}=\operatorname{span}\left\{\sum_{i=1}^{m}\left(\begin{array}{cc}
b_{i}^{2} & -b_{i} \mathrm{a}_{i}^{\top} \\
-b_{i} \mathrm{a}_{i} & \mathrm{a}_{i} \mathrm{a}_{i}^{\top}
\end{array}\right)\right\}, \quad \mathcal{B}_{2}^{\perp}=\operatorname{span}\left\{\sum_{j \in \mathcal{B}}\left(\begin{array}{cc}
0 & -\mathrm{e}_{j}^{\top} \\
-\mathrm{e}_{j} & 2 \mathrm{e}_{j} \mathrm{e}_{j}^{\top}
\end{array}\right)\right\} .
\end{aligned}
$$

Recalling that $\left(\mathcal{C} \mathcal{P}^{n+1}\right)^{*}=\mathcal{C O} \mathcal{P}^{n+1}$ and $\left(\mathcal{S}_{+}^{n+1} \cap \mathcal{N}^{n+1}\right)^{*}=\mathcal{S}_{+}^{n+1}+\mathcal{N}^{n+1}$ (see e.g. 14, Theorem 1.32]), and using the results from the previous subsection, we then get that the dual problems to [22] and [23) for $k \in[1: 2]$ and $i \in[1: 4]$ are respectively

$$
\begin{array}{ll}
\max _{y} & y \\
\text { s.t. } & \left(\begin{array}{cc}
-y & \mathrm{c}^{\top} \\
\mathrm{c} & \mathrm{Q}
\end{array}\right) \in \mathcal{B}_{k}^{\perp}+\mathcal{L}_{i}^{\perp}+\mathcal{C} \mathcal{O} \mathcal{P}^{n+1} \\
\max _{y} & y \\
\text { s.t. } & \left(\begin{array}{cc}
-y & \mathrm{c}^{\top} \\
\mathrm{c} & \mathrm{Q}
\end{array}\right) \in \mathcal{B}_{k}^{\perp}+\mathcal{L}_{i}^{\perp}+\mathcal{S}_{+}^{n+1}+\mathcal{N}^{n+1} .
\end{array}
$$

We now consider the dual to problem $(24)$ for $k \in[1: 2]$. The set $\left\{\mathrm{Y} \in \mathcal{S}^{n+1-m}: \mathrm{RYR}^{\top} \in \mathcal{B}_{k}\right\}$ is a linear subspace with $\left\{\mathrm{Y} \in \mathcal{S}^{n+1-m}: \mathrm{RYR}^{\top} \in \mathcal{B}_{k}\right\}^{\perp}=\mathrm{R}^{\top} \mathcal{B}_{k}^{\perp} \mathrm{R}$. We now let $\mathcal{K}=\left\{\mathrm{Y} \in \mathcal{S}_{+}^{n+1-m}: \mathrm{RYR}^{\top} \in \mathcal{N}^{n+1}\right\}$ and consider the following result, the proof of which is deferred to the Appendix:

Lemma 3 Letting $\mathcal{K}=\left\{\mathrm{Y} \in \mathcal{S}_{+}^{n+1-m}: \mathrm{RYR}^{\top} \in \mathcal{N}^{n+1}\right\}$, we have that $\mathcal{K}$ is a proper cone with $\mathcal{K}^{*}=\mathcal{S}_{+}^{n+1-m}+$ $\mathrm{R}^{\top} \mathcal{N}^{n+1} \mathrm{R}$.

Therefore the dual problem to 24 for $k \in[1: 2]$ is

$$
\begin{array}{ll}
\max _{y} & y \\
\text { s.t. } & \mathrm{R}^{\top}\left(\begin{array}{ll}
0 & c^{\top} \\
c & \mathrm{Q}
\end{array}\right) \mathrm{R}-y \mathrm{R}^{\top}\left(\begin{array}{ll}
1 & 0 \\
0 & 0
\end{array}\right) \mathrm{R} \in \mathrm{R}^{\top} \mathcal{B}_{k}^{\perp} \mathrm{R}+\mathrm{R}^{\top} \mathcal{N}^{n+1} \mathrm{R}+\mathcal{S}_{+}^{n+1-m},
\end{array}
$$

or equivalently

$$
\begin{array}{ll}
\max _{y, \mathrm{Z}} & y \\
\text { s.t. } & \left(\begin{array}{ll}
-y & \mathrm{c} \\
\mathrm{c}^{\top} & \mathrm{Q}
\end{array}\right)-Z \in \mathcal{B}_{k}^{\perp}+\mathcal{N}^{n+1} \\
& \mathrm{R}^{\top} \mathrm{ZR} \in \mathcal{S}_{+}^{n+1-m} .
\end{array}
$$

We can use the following lemma to compare the dual problems with each other: 
Lemma 4 We have

$$
\begin{aligned}
\mathcal{B}_{2}^{\perp} & \subseteq \mathcal{B}_{1}^{\perp} \\
\mathcal{L}_{4}^{\perp} & \subseteq \mathcal{L}_{3}^{\perp} \subseteq \mathcal{L}_{2}^{\perp}=\mathcal{L}_{1}^{\perp} \\
\mathcal{L}_{1}^{\perp}+\mathcal{S}_{+}^{n+1} & =\left\{\mathrm{Z} \in \mathcal{S}^{n+1}: \mathrm{R}^{\top} \mathrm{ZR} \in \mathcal{S}_{+}^{n+1-m}\right\}, \\
\mathcal{B}_{1}^{\perp}+\mathcal{L}_{2}^{\perp}+\mathcal{C O} \mathcal{P}^{n+1} & =\mathcal{B}_{2}^{\perp}+\mathcal{L}_{2}^{\perp}+\mathcal{C} \mathcal{O P}^{n+1}, \\
\mathcal{B}_{1}^{\perp}+\mathcal{L}_{2}^{\perp}+\mathcal{S}_{+}^{n+1}+\mathcal{N}^{n+1} & =\mathcal{B}_{2}^{\perp}+\mathcal{L}_{2}^{\perp}+\mathcal{S}_{+}^{n+1}+\mathcal{N}^{n+1} .
\end{aligned}
$$

Proof. It is trivial to see that $\mathcal{B}_{2}^{\perp} \subseteq \mathcal{B}_{1}^{\perp}$ and from Theorem 1 we have $\mathcal{L}_{4}^{\perp} \subseteq \mathcal{L}_{3}^{\perp} \subseteq \mathcal{L}_{2}^{\perp}=\mathcal{L}_{1}^{\perp}$. From Theorem 1 we also have

$$
\operatorname{cl}\left(\mathcal{L}_{1}^{\perp}+\mathcal{S}_{+}^{n+1}\right)=\left(\mathcal{L}_{1} \cap \mathcal{S}_{+}^{n+1}\right)^{*}=\left(\mathrm{RS}_{+}^{n+1-m} \mathrm{R}^{\top}\right)^{*}=\left\{\mathrm{Z} \in \mathcal{S}^{n+1}: \mathrm{R}^{\top} \mathrm{ZR} \in \mathcal{S}_{+}^{n+1-m}\right\}
$$

and by considering the faces of the psd cone it can be shown that $\left(\mathcal{L}_{1}^{\perp}+\mathcal{S}_{+}^{n+1}\right)$ is in fact closed (see e.g. [19, 20]).

To prove the final two equalities, it is sufficient to show that $\mathcal{B}_{1}^{\perp}+\mathcal{L}_{2}^{\perp}+\mathcal{N}^{n+1}=\mathcal{B}_{2}^{\perp}+\mathcal{L}_{2}^{\perp}+\mathcal{N}^{n+1}$. From Lemma 2 we have that $\mathcal{B}_{1} \cap \mathcal{L}_{2} \cap \mathcal{N}^{n+1}=\mathcal{B}_{2} \cap \mathcal{L}_{2} \cap \mathcal{N}^{n+1}$. Therefore

$$
\operatorname{cl}\left(\mathcal{B}_{1}^{\perp}+\mathcal{L}_{2}^{\perp}+\mathcal{N}^{n+1}\right)=\left(\mathcal{B}_{1} \cap \mathcal{L}_{2} \cap \mathcal{N}^{n+1}\right)^{*}=\left(\mathcal{B}_{2} \cap \mathcal{L}_{2} \cap \mathcal{N}^{n+1}\right)^{*}=\operatorname{cl}\left(\mathcal{B}_{2}^{\perp}+\mathcal{L}_{2}^{\perp}+\mathcal{N}^{n+1}\right) .
$$

The proof is then completed by noting that as the sets $\mathcal{B}_{1}, \mathcal{B}_{2}, \mathcal{L}_{2}$ and $\mathcal{N}^{n+1}$ are all polyhedra, then so are their Minkowski sums, and in particular this means that these sums are closed.

\subsection{Monotonicity of the duality gaps}

We can now prove monotonicity of the duality gap in the number $i$ of our dual problems, thereby giving a complete relation of all considered reformulations and relaxations from a duality perspective.

Theorem 7 Consider the dual problems (25) or (26). As i increases, so does the duality gap. Therefore picking $i=1$ for problem (22) (and its relaxation (24) not only gives the smallest problems in terms of number of variables and constraints (and order of psd constraints for the relaxation), but also gives the smallest duality gap. For both of these problems, with $i=1$, the feasible sets of their primal and dual problems are independent of $k$. However picking $k=2$ will reduce the number of constraints in the primal problems, and the number of variables in the dual problems (when compared to $k=1$ ).

Proof. From Lemma 4, we see that as $i$ increases the feasible sets of the dual problems (25) or 26) shrink (or at least remain the same). This means that the duality gap is monotonically increasing with $i$.

The previous result suggests that for the completely positive problem, we should always use $(22)$ with $k=2$ and $i=1$, whilst for the relaxation, we should always use 24 with $k=2$. This will provide the smallest problems and duality gaps. As an example of a duality gap occurring see below:

Example 2 Consider the following problem with no binary variables

$$
\min _{\mathrm{x}}\left\{2 x_{2}-2 x_{1} x_{2}: x_{1}=1, \mathrm{x} \in \mathbb{R}_{+}^{2}\right\}
$$

i.e., $n=2, m=b_{1}=1, \mathrm{a}=\left(\begin{array}{l}1 \\ 0\end{array}\right), \mathrm{c}=\left(\begin{array}{l}0 \\ 1\end{array}\right), \mathrm{Q}=\left(\begin{array}{cc}0 & -1 \\ -1 & 0\end{array}\right)$ and opt $28=0$. For $i=3$, the completely positive reformulation 22 and its dual 25 are respectively

$$
\begin{aligned}
& \min _{\mathrm{x}, \mathrm{X}}\left\{2 x_{2}-2 x_{12}: x_{1}=1, x_{11}=1,\left(\begin{array}{ll}
1 & \mathbf{x}^{\top} \\
\mathbf{x} & \mathbf{x}
\end{array}\right) \in \mathcal{C} \mathcal{P}^{3}\right\} \\
& \max _{u, v, y}\left\{y:\left(\begin{array}{ccc}
-y & 0 & 1 \\
0 & 0 & -1 \\
1 & -1 & 0
\end{array}\right)+u\left(\begin{array}{ccc}
2 & -1 & 0 \\
-1 & 0 & 0 \\
0 & 0 & 0
\end{array}\right)+v\left(\begin{array}{ccc}
-1 & 0 & 0 \\
0 & 1 & 0 \\
0 & 0 & 0
\end{array}\right) \in \mathcal{C O} \mathcal{P}^{3}\right\} .
\end{aligned}
$$

It can then be shown that

$$
\text { feas }(29)=\left\{(\mathrm{x}, \mathrm{X}) \in \mathbb{R}_{+}^{2} \times \mathcal{S}^{2}: x_{1}=1, x_{11}=1, x_{12}=x_{2}, x_{2}^{2} \leq x_{22}\right\} \quad \text { and } \quad \text { feas } 300 .
$$


Therefore opt $(30)=-\infty<0=$ opt $(29)=$ opt 28$)$. But when we use the dual 25$]$ with $i=1$, the duality gap is closed and strong duality obtains. Indeed, the matrix $Z=\left(\begin{array}{ccc}-y & 0 & 1 \\ 0 & 0 & -1 \\ 1 & -1 & 0\end{array}\right)$ with $y=0$ already satisfies $\mathrm{R}^{\top} \mathrm{ZR}=\mathrm{O}$ (if $\mathrm{x}_{0}=(1,1)^{\top}$ and $\mathrm{a}_{2}=(0,1)^{\top}$ are chosen for $\mathrm{R}$ ), so we get $\mathrm{Z} \in \mathcal{L}_{1}^{\perp} \subset \mathcal{L}_{1}^{\perp}+\mathcal{C O} \mathcal{P}^{3}$, hence $y=0$ is feasible (and optimal) to 25 with the choice $i=1$, and full strong duality holds.

\subsection{Conditions for zero duality gap}

When can we guarantee that there will be no duality gap? One simple case when this can be guaranteed is covered in the following theorem.

Theorem 8 If the polyhedron $\mathcal{Z}=\left\{\mathrm{x} \in \mathbb{R}_{+}^{n}: \mathrm{a}_{j}^{\top} \mathrm{x}=b_{j}, j \in[1: m]\right\}$ is bounded then Slater's condition holds for problems 25), 26) and (27) (for all $k \in[1: 2], i \in[1: 4]$ ).

Proof. If $\mathcal{Z}$ is bounded then for $B=\emptyset$, the feasible sets of (22), (23) and (24) are bounded and nonempty. From Corollary 1 we then get that, for $B=\emptyset$, Slater's condition holds for problems (25), (26) and (27). For the case when $B \neq \emptyset$, we are simply increasing the size of the feasible sets for (25), (26) and (27), and thus Slater's condition continues to hold.

If $\mathcal{Z}$ is unbounded then checking Slater's condition is somewhat more complicated, with the proof of the next result being left to the appendix:

Theorem 9 Assume that the polyhedron $\mathcal{Z}=\left\{\mathrm{x} \in \mathbb{R}_{+}^{n}: \mathrm{a}_{i}^{\top} \mathrm{x}=b_{i}, i \in[1: m]\right\}$ is unbounded, and let $J$ be the unbounded indices, i.e.

$$
\emptyset \neq J:=\left\{j \in[1: n]: \sup \left\{x_{j}: x \in \mathcal{Z}\right\}=\infty\right\} \subseteq[1: n] \backslash B .
$$

Let $\widetilde{\mathrm{Q}}$ be the principal submatrix of $\mathrm{Q}$ corresponding to the indices $J$ and let $\widetilde{\mathrm{a}}_{j}$ be the subvector of $\mathrm{a}_{j}$ corresponding to the indices $J$.

Then Slater's condition holds for problem (25) (for all $k \in[1: 2]$, ie[1:4]) if and only if $\mathbf{z}^{\top} \widetilde{\mathbf{Q} z}>0$ for all $\mathrm{z} \in \mathbb{R}_{+}^{|J|} \backslash\{\mathrm{o}\}$ with $\widetilde{\mathrm{a}}_{j}^{\top} \mathrm{z}=0$ for all $j \in[1: m]$.

Slater's condition holds for problems (26) and 27) (for all $k \in[1: 2], i \in[1: 4]$ ) if and only if

$$
\widetilde{\mathrm{Q}} \in \operatorname{int}\left(\mathcal{N}^{|J|}+\mathcal{S}_{+}^{|J|}+\operatorname{span}\left\{\widetilde{\mathbf{a}}_{j} \widetilde{\mathbf{a}}_{j}^{\top}: j \in[1: m]\right\}\right) .
$$

Note that Slater's condition holding is independent of the set of binary variables, $B$. We also note that a simple sufficient condition for Slater's condition holding is that $\widetilde{Q} \in \operatorname{int}\left(\mathcal{N}^{|J|}+\mathcal{S}_{+}^{|J|}\right)$.

The primal problems not being strictly feasible means that the optimal values for the dual problems may not be attained, and we now consider a simple example of this happening:

Example 3 Consider the problem

$$
\min _{x}\left\{x^{2}: x=1, x \geq 0\right\}
$$

i.e., $1=n=\mathrm{Q}=m=\mathrm{a}_{1}=b_{1}=$ opt (31) and $\mathrm{c}=0$. For $i=4$, the completely positive reformulation to this 22 and its dual 25 are respectively

$$
\begin{aligned}
& \min _{v, x}\left\{v: v-2 x+1=0,\left(\begin{array}{ll}
1 & x \\
x & v
\end{array}\right) \in \mathcal{C P}^{2}\right\}, \\
& \max _{y, z}\left\{y:\left(\begin{array}{cc}
-y & 0 \\
0 & 1
\end{array}\right)+z\left(\begin{array}{cc}
1 & -1 \\
-1 & 1
\end{array}\right) \in \mathcal{C O} \mathcal{P}^{2}\right\} .
\end{aligned}
$$

It can then be shown that feas 32$\}=\{(1,1)\}$ and

$$
\text { feas } 33\}=\left\{(y, z) \in \mathbb{R}^{2}:-1 \leq z \leq 0, y \leq z\right\} \cup\left\{(y, z) \in \mathbb{R}^{2}: z \geq 0, y \leq \frac{z}{1+z}\right\} \text {. }
$$

Therefore $1=$ opt $(31)=$ opt $(32)=$ opt $(33)$, but the optimal solution to 33$)$ is not attained. 


\section{Conclusion and outlook}

In this paper we have tried to unify all existing alternative CP reformulations and relaxations of mixed-binary QPs, complementing them with several novel approaches. We discussed in detail the duality requirements which ensure attainability and/or zero duality gap (strong duality) for these reformulations and relaxations, offering a complete relationship of their respective duality gaps and conditions for strong duality.

The following research questions could be interesting projects for the future:

1. In this paper we did not consider sparsity, however this can make a significant difference in the running time of solvers. In particular, we could ask what sparsity is lost when finding the orthogonal complement $\left\{a_{1}, \ldots, a_{m}\right\}^{\perp}$ in the construction of R. A starting point for the investigation of this would be the paper [17]

2. Can we relax the nonnegative constraints by aggregating them (e.g. instead of requiring $X_{i j} \geq 0$ for all $i, j$, we let $\mathcal{I}_{1}, \ldots, \mathcal{I}_{p} \subseteq[1: n]^{2}$ and require $0 \leq \sum_{(i, j) \in \mathcal{I}_{k}} X_{i j}$ for all $\left.k\right)$ ?

3. If we use approximation hierarchies like that of Parrilo, can we use the linear constraints in the original problem to reduce the order of the PSD matrices in this approximation (in a similar manner to what we did for the zero level approximation where $\mathcal{C} \mathcal{P}^{d}$ is approximated by $\left.\mathcal{S}_{+}^{d} \cap \mathcal{N}^{d}\right)$ ?

\section{Acknowledgements}

The authors would like to thank the anonymous handling Associate Editor and two anonymous referees for their very useful comments and suggestions which helped to improve our paper significantly. This research benefited from the support of the "FMJH Program Gaspard Monge in Optimization and Operations Research", and from the support to this program by EDF.

\section{References}

1. N. Arima, S. Kim, and M. Kojima. Simplified copositive and Lagrangian relaxations for linearly constrained quadratic optimization problems in continuous and binary variables. Pacific J. Optimiz. 10: 437-451 (2014).

2. A. Berman, Cones, Matrices and Mathematical Programming. Lecture Notes in Economics and Mathematical Systems 79 (1973), Springer Verlag.

3. A. Berman and N. Shaked-Monderer, Completely positive matrices. World Scientific Publ, River Edge, NJ, 2003.

4. I.M. Bomze, Copositive optimization - recent developments and applications. European J. Oper. Res. 216: 509-520 (2012).

5. I.M. Bomze, Copositive relaxation beats Lagrangian dual bounds in quadratically and linearly constrained QPs. $S I A M$ J. Optimiz. 25 (2015): 1249-1275.

6. I.M. Bomze, M. Dür, E. de Klerk, C. Roos, A.J. Quist and T. Terlaky, On copositive programming and standard quadratic optimization problems, Journal of Global Optimization 18: 301-320 (2000).

7. I.M. Bomze and F. Jarre, A note on Burers copositive representation of mixed-binary QPs. Optimization Letters 4: 465-472 (2010).

8. I.M. Bomze, W. Schachinger, and R. Ullrich. New lower bounds and asymptotics for the cp-rank. SIAM J. Matrix Anal. Appl., 36(1): 20-37 (2015).

9. I.M. Bomze, W. Schachinger, and G. Uchida. Think co(mpletely)positive ! Matrix properties, examples and a clustered bibliography on copositive optimization. J. Glob. Optimiz. 52: 423-445 (2012).

10. S. Burer, On the copositive representation of binary and continuous nonconvex quadratic programs. Mathematical Programming 120(2): 479-495 (2009).

11. S. Burer, Optimizing a polyhedral-semidefinite relaxation of completely positive programs. Mathematical Programming Computation 2(1): 1-19 (2010).

12. S. Burer, Copositive programming. In Handbook of Semidefinite, Cone and Polynomial Optimization: Theory, Algorithms, Software and Applications (M.F.Anjos and J.B.Lasserre, eds.), International Series in Operations Research and Management Science, Springer New York (2012), pp. 201-218.

13. S. Burer, A gentle, geometric introduction to copositive optimization. Mathematical Programming 151 (2015): 89-116.

14. P.J.C. Dickinson, The copositive cone, the completely positive cone and their generalisations, PhD thesis, University of Groningen, 2013.

15. P.J.C. Dickinson and L. Gijben, On the computational complexity of membership problems for the completely positive cone and its dual, Computational Optimization and Applications, 57(2): 403-415 (2014).

16. M. Dür, Copositive Programming — a Survey. In Recent Advances in Optimization and its Applications in Engineering (M.Diehl, F.Glineur, E.Jarlebring and W.Michiels, eds.), Springer Berlin (2010), pp. 3-20.

17. J.R. Gilbert and M.T. Heath, Computing a sparse basis for the null space, SIAM Journal on Algebraic Discrete Methods, 8(3): 446-459 (1987).

18. K.G. Murty and S.N. Kabadi, Some NP-complete problems in quadratic and nonlinear programming, Mathematical Programming 39(2): 117-129 (1987).

19. G. Pataki, The geometry of semidefinite programming, Handbook of Semidefinite Programming: Theory, Algorithms, and Applications, H. Wolkowicz, R. Saigal and L. Vandenberghe (Eds.), Springer, 2000, 29-65. 
20. G. Pataki, On the closedness of the linear image of a closed convex cone, Mathematics of Operations Research, 32(2): 395-412 (2007).

21. N. Shaked-Monderer, A. Berman, I.M.Bomze, F. Jarre, and W. Schachinger, New results on the cp rank and related properties of co(mpletely )positive matrices. Linear Multilinear Algebra, 63(2): 384-396 (2015).

22. N.Z. Shor, Quadratic optimization problems. Izv. Akad. Nauk SSSR Tekhn. Kibernet. 222(1): 128-139 (1987).

23. L.N. Trefethen and D. Bau III, Numerical linear algebra. Vol. 50. SIAM, Philadelphia PA, 1997.

\section{Appendix}

Proof of Theorem 6. The characterizations of int $\left(\mathcal{L}^{\perp}+\mathcal{K}^{*}\right)$ in Theorem 6 follow trivially from the definition of the interior and from $\mathcal{K}^{*}$ being a convex cone with nonempty interior (see e.g. [2, Theorem 2.3]).

If Slater's condition, 1, holds then we can approach the optimal value to (17) from the interior, and thus the closure is not necessary (i.e., for all $y \in$ feas $[17)$ and all $\varepsilon \in] 0,1]$ we have $\mathrm{q}-(y+\varepsilon(\widehat{y}-y)) \mathrm{d}_{0} \in \operatorname{int}\left(\mathcal{L}^{\perp}+\mathcal{K}^{*}\right)$, and thus $y+\varepsilon(\widehat{y}-y) \in$ feas (18)). This implies that opt (18) $=\operatorname{opt}(17)=$ opt (15), with the last equality following from Theorem 4 .

We are thus left to prove the equivalence relations. In doing this we will repeatedly use the result that for a closed convex pointed cone $\mathcal{C} \subseteq \mathbb{R}^{n}$ we have $\operatorname{int} \mathcal{C}^{*}=\left\{z \in \mathbb{R}^{n}:\langle x, z\rangle>0\right.$ for all $x \in \mathcal{C} \backslash\{$ o $\left.\}\right\}$ (see e.g. 2, Equation (2.1)]). We split the proof of the equivalence relations into four parts:

1 일

$$
\begin{aligned}
\left\{\mathrm{q} \in \mathbb{R}^{n}\right. & \left.:\langle\mathrm{q}, \mathrm{u}\rangle>0 \text { for all } \mathrm{u} \in \mathcal{L} \cap \mathcal{K} \backslash\{\mathrm{o}\} \text { s.t. }\left\langle\mathrm{d}_{0}, \mathrm{u}\right\rangle=0\right\} \\
& =\operatorname{int}\left(\left\{\mathrm{u} \in \mathcal{L} \cap \mathcal{K}:\left\langle\mathrm{d}_{0}, \mathrm{u}\right\rangle=0\right\}^{*}\right) \\
& =\operatorname{int}\left(\operatorname{cl}\left(\operatorname{span}\left\{\mathrm{d}_{0}\right\}+\mathcal{L}^{\perp}+\mathcal{K}^{*}\right)\right) \\
& =\operatorname{int}\left(\operatorname{span}\left\{\mathrm{d}_{0}\right\}+\left(\mathcal{L}^{\perp}+\mathcal{K}^{*}\right)\right) \\
& =\operatorname{span}\left\{\mathrm{d}_{0}\right\}+\operatorname{int}\left(\mathcal{L}^{\perp}+\mathcal{K}^{*}\right) \\
& =\left\{\mathrm{q} \in \mathbb{R}^{n}: \exists y \in \mathbb{R} \text { s.t. } \mathrm{q}-y \mathrm{~d}_{0} \in \operatorname{int}\left(\mathcal{L}^{\perp}+\mathcal{K}^{*}\right)\right\} .
\end{aligned}
$$

2 $\Rightarrow$ 4: Suppose for the sake of contradiction that statement 2 is true but there exists $\mu \in \mathbb{R}$ such that the set $\{x \in$ feas $[15):\langle q, x\rangle \leq \mu\}$ is not compact. This set is the intersection of closed sets and thus is itself closed. Therefore it must be unbounded, i.e. for all $i \in \mathbb{N}$ there exists $\mathrm{x}_{i} \in$ feas 15 such that $\left\|\mathrm{x}_{i}\right\|_{2}>i$ and $\left\langle\mathrm{q}, \mathrm{x}_{i}\right\rangle \leq \mu$.

For all $i$, letting $\mathbf{u}_{i}=\frac{1}{\left\|x_{i}\right\|_{2}} x_{i}$ we have $\left\|\mathbf{u}_{i}\right\|_{2}=1$ and thus the sequence $\left\{\mathbf{u}_{i}: i \in \mathbb{N}\right\}$ is limited to a compact set. Therefore there is a limiting subsequence tending towards a limiting point $u$.

For all $i$ we have $\mathrm{u}_{i} \in \mathcal{L} \cap \mathcal{K}$ and $\left\|\mathrm{u}_{i}\right\|_{2}=1$, and as $\mathcal{L} \cap \mathcal{K}$ is closed this implies that $\mathrm{u} \in \mathcal{L} \cap \mathcal{K} \backslash\{\mathrm{o}\}$. We also have $\left\langle\mathrm{d}_{0}, \mathrm{u}\right\rangle=\lim _{i \rightarrow \infty} \frac{1}{\left\|\mathrm{x}_{i}\right\|_{2}}\left\langle\mathrm{~d}_{0}, \mathrm{x}_{i}\right\rangle=0$ and $\langle\mathrm{q}, \mathrm{u}\rangle \leq \lim _{i \rightarrow \infty} \frac{\mu}{\left\|x_{i}\right\|_{2}}=0$. This gives the contradiction that statement 2 does not hold.

4 feas $\sqrt{15} \neq \emptyset \Rightarrow$ 3: This follows from considering an arbitrary $x \in$ feas $(15)$ and letting $\mu=\langle q, x\rangle$.

$3 \Rightarrow$ 2: Suppose there exists $\mu \in \mathbb{R} \cup\{\infty\}$ such that the set $\{x \in$ feas 15$\rangle:\langle q, x\rangle \leq \mu\}$ is nonempty and compact, and additionally suppose for the sake of contradiction that there exists a $u \in \mathcal{L} \cap \mathcal{K} \backslash\{\mathrm{o}\}$ such that $\langle\mathrm{q}, \mathrm{u}\rangle \leq$ $0=\left\langle\mathrm{d}_{0}, \mathrm{u}\right\rangle$. Then there exists $\mathrm{v} \in$ feas $(15)$ such that $\langle\mathrm{q}, \mathrm{v}\rangle \leq \mu$, and for all $\lambda \geq 0$ we have $\mathrm{v}+\lambda \mathrm{u} \in$ feas $(15)$ and $\langle\mathrm{q}, \mathrm{v}+\lambda \mathrm{u}\rangle \leq\langle\mathrm{q}, \mathrm{v}\rangle \leq \mu$, which is a contradiction.

Proof of Lemma 3. To establish Lemma 3, first note that $\mathcal{K}$ is a closed convex pointed cone. For $\varepsilon \in] 0,1]$, letting $\mathrm{Y}=\varepsilon \mathbf{l}+(1-\varepsilon) \mathrm{e}_{0} \mathrm{e}_{0}^{\top}$, we have $\mathrm{Y} \in \operatorname{int} \mathcal{S}_{+}^{n+1-m}$ and $\mathrm{RYR}^{\top}=\varepsilon \mathrm{R}^{\top} \mathrm{R}+(1-\varepsilon)\left(\begin{array}{c}1 \\ \mathrm{x}_{0}\end{array}\right)\left(\begin{array}{c}1 \\ \mathrm{x}_{0}\end{array}\right)^{\top}$ which is strictly positive for $\varepsilon>0$ small enough. Therefore $Y \in \operatorname{int} \mathcal{K}$ for $\varepsilon>0$ small enough, completing the proof that $\mathcal{K}$ is a proper cone. The remainder of the proof then follows from the following equalities:

$$
\left(\mathcal{S}_{+}^{n+1-m}\right)^{*}=\mathcal{S}_{+}^{n+1-m},
$$




$$
\begin{aligned}
\left(\mathrm{R}^{\top} \mathcal{N}^{n+1} \mathrm{R}\right)^{*} & =\left\{\mathrm{Y} \in \mathcal{S}^{n+1-m}:\left\langle\mathrm{Y}, \mathrm{R}^{\top} \mathrm{XR}\right\rangle \geq 0 \text { for all } \mathrm{X} \in \mathcal{N}^{n+1}\right\} \\
& =\left\{\mathrm{Y} \in \mathcal{S}^{n+1-m}:\left\langle\mathrm{RYR}^{\top}, \mathrm{X}\right\rangle \geq 0 \text { for all } \mathrm{X} \in \mathcal{N}^{n+1}\right\} \\
& =\left\{\mathrm{Y} \in \mathcal{S}^{n+1-m}: \mathrm{RYR}^{\top} \in \mathcal{N}^{n+1}\right\}, \\
\left\{\mathrm{Y} \in \mathcal{S}^{n+1-m}: \mathrm{RYR}^{\top} \in \mathcal{N}^{n+1}\right\}^{*} & =\left(\mathrm{R}^{\top} \mathcal{N}^{n+1} \mathrm{R}\right)^{* *}=\operatorname{cl}\left(\mathrm{R}^{\top} \mathcal{N}^{n+1} \mathrm{R}\right)=\mathrm{R}^{\top} \mathcal{N}^{n+1} \mathrm{R}, \\
\mathcal{K}^{*} & =\operatorname{cl}\left(\left(\mathcal{S}_{+}^{n+1-m}\right)^{*}+\left\{\mathrm{Y} \in \mathcal{S}^{n+1-m}: \mathrm{RYR}^{\top} \in \mathcal{N}^{n+1}\right\}^{*}\right) \\
& =\operatorname{cl}\left(\mathcal{S}_{+}^{n+1-m}+\mathrm{R}^{\top} \mathcal{N}^{n+1} \mathrm{R}\right) \\
& =\mathcal{S}_{+}^{n+1-m}+\mathrm{R}^{\top} \mathcal{N}^{n+1} \mathrm{R} .
\end{aligned}
$$

The equalities (34) and (35) follow from the well known result that both $\mathcal{S}_{+}^{n+1-m}$ and $\mathcal{N}^{n+1}$ are self-dual (see e.g. 14, Theorem 1.32]). The equalities in (36) follow from (35), 2, Corollary 2.1] and $\mathcal{N}^{n+1}$ being a polyhedron implying that $\mathrm{R}^{\top} \mathcal{N}^{n+1} \mathrm{R}$ is a polyhedron. Finally, the equality (37) follows from [2, Corollary 2.2] and equality (38) follows from [14, Corollary 1.12].

Proof of Theorem 9. First we consider problem (25) for arbitrary $k \in[1: 2], i \in[1: 4]$. From Theorem 6 we have that Slater's condition holds if and only if

$$
\left\langle\left(\begin{array}{ll}
0 & c^{\top} \\
c & Q
\end{array}\right),\left(\begin{array}{cc}
x_{0} & x^{\top} \\
x & X
\end{array}\right)\right\rangle>0 \quad \text { for all }\left(\begin{array}{cc}
x_{0} & x^{\top} \\
x & X
\end{array}\right) \in \mathcal{B}_{k} \cap \mathcal{L}_{i} \cap \mathcal{C} \mathcal{P}^{n+1} \backslash\{O\} \text { such that } x_{0}=0 .
$$

or equivalently

$$
\langle\mathrm{Q}, \mathrm{X}\rangle>0 \quad \text { for all } \mathrm{X} \in \mathcal{S}^{n} \backslash\{\mathrm{O}\} \text { such that }\left(\begin{array}{ll}
0 & \mathrm{o}^{\top} \\
\mathrm{o} & \mathrm{X}
\end{array}\right) \in \mathcal{B}_{k} \cap \mathcal{L}_{i} \cap \mathcal{C} \mathcal{P}^{n+1} .
$$

From Proposition 1. this condition is independent of $k$ and $l$, and from Lemma 1 it can be shown that $\left(\begin{array}{ll}0 & o^{\top} \\ \circ & X\end{array}\right) \in \mathcal{B}_{k} \cap \mathcal{L}_{i} \cap \mathcal{C} \mathcal{P}^{n+1}$ if and only if

$$
\begin{aligned}
X_{j k} & =0 \text { for all } j \in[1: n] \backslash J, k \in[1: n], \\
\widetilde{\mathrm{X}} & \in \operatorname{conv}\left\{\mathrm{vv}^{\top}: \mathrm{v} \in \mathbb{R}_{+}^{|J|}, \widetilde{\mathrm{a}}_{i}^{\top} \mathbf{v}=0 \text { for all } i \in[1: m]\right\},
\end{aligned}
$$

where $\widetilde{X}$ is the principal submatrix of $\mathrm{X}$ corresponding to the indices $J$. This completes the proof for problem (25).

We next consider problem (26) for arbitrary $k \in[1: 2], i \in[1: 4]$. Similarly to before, from Theorem 6 we have that Slater's condition holds if and only if

$$
\langle\mathrm{Q}, \mathrm{X}\rangle>0 \quad \text { for all } \mathrm{X} \in \mathcal{S}^{n} \backslash\{\mathrm{O}\} \text { such that }\left(\begin{array}{ll}
0 & \mathrm{o}^{\top} \\
\mathrm{o} & \mathrm{X}
\end{array}\right) \in \mathcal{B}_{k} \cap \mathcal{L}_{i} \cap \mathcal{S}_{+}^{n+1} \cap \mathcal{N}^{n+1} .
$$

From Proposition 1 this condition is independent of $k$ and $i$, and from Lemma 1 it can be shown that $\left(\begin{array}{ll}0 & 0^{\top} \\ 0 & X\end{array}\right) \in \mathcal{L}_{2} \cap \mathcal{N}^{n+1}$ if and only if

$$
\begin{aligned}
X_{j k} & =0 \text { for all } j \in[1: n] \backslash J, k \in[1: n], \\
\widetilde{\mathrm{X}} & \in \mathcal{N}^{|J|} \\
\widetilde{\mathrm{X}} \widetilde{\mathrm{a}}_{j} & =0 \text { for all } j \in[1: m],
\end{aligned}
$$

and we then additionally have $\left(\begin{array}{ll}0 & \mathrm{o}^{\top} \\ \mathrm{o} & \mathrm{X}\end{array}\right) \in \mathcal{B}_{k}$. Therefore $\left(\begin{array}{ll}0 & \mathrm{o}^{\top} \\ 0 & \mathrm{X}\end{array}\right) \in \mathcal{B}_{k} \cap \mathcal{L}_{i} \cap \mathcal{S}_{+}^{n+1} \cap \mathcal{N}^{n+1}$ if and only if

$$
X_{j k}=0 \text { for all } j \in[1: n] \backslash J, k \in[1: n],
$$

$\left\langle\widetilde{\mathbf{a}}_{j} \widetilde{\mathrm{a}}_{j}^{\top}, \widetilde{\mathrm{X}}\right\rangle=0$ for all $j \in[1: m]$,

$$
\widetilde{\mathrm{X}} \in \mathcal{S}_{+}^{|J|} \cap \mathcal{N}^{|J|}
$$


which completes the proof for problem 26 .

Finally we consider problem (27) for all $k \in[1: 2]$. From Theorem 6 we have that Slater's condition holds if and only if

$$
\begin{aligned}
& \left\langle\mathrm{R}^{\top}\left(\begin{array}{ll}
0 & c^{\top} \\
c & \mathrm{Q}
\end{array}\right) \mathrm{R}, \mathrm{Y}\right\rangle>0 \text { for all } \mathrm{Y} \in \mathcal{S}_{+}^{n+1-m} \backslash\{\mathrm{O}\} \text { s.t. } \mathrm{RYR}^{\top} \in \mathcal{B}_{k}, \mathrm{RYR}^{\top} \in \mathcal{N}^{n+1}, \\
& \left\langle R^{\top}\left(\begin{array}{ll}
1 & 0^{\top} \\
0 & 0
\end{array}\right) R, Y\right\rangle=0
\end{aligned}
$$

or equivalently

$$
\left\langle\left(\begin{array}{ll}
0 & c^{\top} \\
c & Q
\end{array}\right),\left(\begin{array}{cc}
x_{0} & \mathbf{x}^{\top} \\
\mathrm{x} & \mathrm{x}
\end{array}\right)\right\rangle>0 \text { for all }\left(\begin{array}{cc}
x_{0} & \mathrm{x}^{\top} \\
\mathrm{x} & \mathrm{x}
\end{array}\right) \in \mathcal{B}_{k} \cap\left(\mathrm{R \mathcal {S } _ { + } ^ { n + 1 - m }} \mathrm{R}^{\top}\right) \cap \mathcal{N}^{n+1} \backslash\{\mathrm{O}\} \text { s.t. } x_{0}=0 .
$$

From Theorem 1, this is equivalent to the previous case for $i=1$. 